\title{
Cerebellar Neurodegeneration and Neuronal Circuit Remodeling in Golgi pH Regulator- Deficient Mice
}

\author{
Yu-shin Sou, ${ }^{1}$ Soichiro Kakuta, ${ }^{2,3}$ (1) Yuji Kamikubo, ${ }^{4}$ Kazue Niisato, ${ }^{4}$ Takashi Sakurai, ${ }^{4}$ Laxmi Kumar \\ Parajuli, ${ }^{1}$ Isei Tanida, ${ }^{1}$ Hiromitsu Saito, ${ }^{5}$ Noboru Suzuki, ${ }^{5}$ Kenji Sakimura, ${ }^{6}$ Yusuke Maeda, ${ }^{7}$ (1)Taroh \\ Kinoshita, ${ }^{7}$ Yasuo Uchiyama, ${ }^{3}$ and Masato Koike ${ }^{1,8}$
}

\author{
https://doi.org/10.1523/ENEURO.0427-18.2019
}

\begin{abstract}
${ }^{1}$ Department of Cell Biology and Neuroscience, Juntendo University Graduate School of Medicine, Bunkyo, Tokyo 113-8421, Japan, ${ }^{2}$ Laboratory of Morphology and Image Analysis, Research Support Center, Juntendo University Graduate School of Medicine, Bunkyo, Tokyo 113-8421, Japan, ${ }^{3}$ Department of Cellular and Molecular

Neuropathology, Juntendo University Graduate School of Medicine, Bunkyo, Tokyo 113-8421, Japan, ${ }^{4}$ Department of Pharmacology, Juntendo University Graduate School of Medicine, Bunkyo, Tokyo 113-8421, Japan, ${ }^{5}$ Functional Genomics Institute, Life Science Research Center, Mie University, Tsu, Mie 514-8507, Japan, ${ }^{6}$ Department of Cellular Neurobiology, Brain Research Institute, Niigata University, Niigata 951-8585, Japan, ${ }^{7}$ Research Institute for Microbial Diseases, Osaka University, Suita, Osaka 565-0871, Japan, and ${ }^{8}$ Advanced Research Institute for Health Science, Juntendo University, Bunkyo, Tokyo 113-8421, Japan
\end{abstract}

\begin{abstract}
The Golgi apparatus plays an indispensable role in posttranslational modification and transport of proteins to their target destinations. Although it is well established that the Golgi apparatus requires an acidic luminal pH for optimal activity, morphological and functional abnormalities at the neuronal circuit level because of perturbations in Golgi pH are not fully understood. In addition, morphological alteration of the Golgi apparatus is associated with several neurodegenerative diseases, including Parkinson's disease, Alzheimer's disease, and amyotrophic lateral sclerosis. Here, we used anatomical and electrophysiological approaches to characterize morphological and functional abnormalities of neuronal circuits in Golgi $\mathrm{pH}$ regulator (GPHR) conditional knock-out mice. Purkinje cells (PCs) from the mutant mice exhibited vesiculation and fragmentation of the Golgi apparatus, followed by axonal degeneration and progressive cell loss. Morphological analysis provided evidence for the disruption of basket cell (BC) terminals around PC soma, and electrophysiological recordings showed selective loss of large amplitude responses, suggesting BC terminal disassembly. In addition, the innervation of mutant PCs was altered such that climbing fiber (CF) terminals abnormally synapsed on the somatic spines of mutant PCs in the mature cerebellum. The combined results describe an essential role for luminal acidification of the Golgi apparatus in maintaining proper neuronal morphology and neuronal circuitry.
\end{abstract}

Key words: cerebellar circuit; Golgi apparatus; Golgi fragmentation; Golgi pH regulator; neurodegeneration; Purkinje cell

\section{Significance Statement}

The Golgi luminal acidic condition is essential for normal activity of the Golgi apparatus and its dysregulation contributes to several human diseases. In neurons, however, pharmacological tools cannot be used for in vivo studies because they lack specificity. Here, the in vivo role of Golgi luminal acidification by inactivation of the Golgi pH regulator (GPHR) in cerebellar neurons is reported. The GPHR is indispensable for neuronal survival, normal Golgi morphology and axonal integrity. Intriguingly, after formation of Pinceau structures, basket cells (BCs) exhibited significant loss of function, resulting in an alteration of neuronal circuitry around Purkinje cells (PCs). These results highlight an essential role of the Golgi luminal pH in the maintenance of gross axonal morphology and subcellular structures in PCs. 


\section{Introduction}

The flattened, cisternal structure of the Golgi apparatus is essential for ensuring that proteins are correctly glycosylated and sorted to the appropriate destination (Klumperman, 2011; Papanikou and Glick, 2014). The optimal activity of intracellular organelles, such as the Golgi apparatus, is strictly regulated by the acidic $\mathrm{pH}$ of the lumen (Weisz, 2003). Perturbation of the luminal $\mathrm{pH}$ not only has marked effects on posttranslational modification and transport of proteins but also causes morphological abnormalities in the Golgi apparatus (Palokangas et al., 1994; Kellokumpu et al., 2002). Similar morphologic abnormalities have also been observed in cerebellar Purkinje cells (PCs; Baloyannis, 2014) and motor neurons in patients with neurodegenerative disorders (Gonatas et al., 1992; Mourelatos et al., 1996; Haase and Rabouille, 2015; Ayala and Colanzi, 2017). However, the causative link between impaired luminal acidification of the Golgi apparatus, fragmentation of the Golgi apparatus and neurodegeneration remains unresolved.

Several hypotheses have been proposed to explain how fragmentation of the Golgi apparatus can cause neurodegeneration. Some of these include microtubule depolymerization, pathogenic protein misfolding, mislocalization of aggregate-prone proteins and phosphorylation of GRASP65, a structural protein of the Golgi apparatus (Alvarez-Miranda et al., 2015). In addition to these explanations, we hypothesized that the impairment of luminal acidification of the Golgi apparatus causes fragmentation, which results in neurodegeneration. Ubiquitin protein ligase E3A (Ube3a), a causative gene product of Angelman's syndrome, is involved in the acidification of the Golgi lumen (Condon et al., 2013). Interestingly, the Golgi-associated protein, ATP6V0A2, is involved in congenital disorders of glycosylation (CDG) because of a $\mathrm{pH}$ imbalance in the Golgi apparatus (Kornak et al., 2008; Demaegd et al., 2013). Additionally, patients with several types of CDG as a result of mutations in genes encoding Golgi-associated proteins show cerebellar atrophy and ataxia (Climer et al., 2015).

Received November 1, 2018; accepted May 12, 2019; First published May 22, 2019

The authors declare no competing financial interests.

Author contributions: Y.-s.S. and M.K. designed research; Y.-s.S., S.K., Y.K., K.N., T.S., L.K.P., and M.K. performed research; Y.-s.S., I.T., H.S., N.S., K.S., Y.M., T.K., and Y.U. contributed unpublished reagents/analytic tools; Y.-s.S., S.K., Y.K., K.N., T.S., and M.K. analyzed data; Y.-s.S., L.K.P., Y.M., T.K., Y.U., and M.K. wrote the paper.

This work was supported by Grants-in-Aid for Scientific Research (15K18961 and 19K07276 to Y.-s.S.; $17 \mathrm{~K} 08522$ and $15 \mathrm{H} 01388$ to M.K.) from the Japan Society for the Promotion of Science (JSPS) and by the Private School Branding Project from the Ministry of Education, Culture, Sports, Science and Technology of Japan (MEXT; M.K.).

Acknowledgements: We thank Dr. Hiroyuki Hioki, Dr. Takehiko Sunabori, and Dr. Kenta Yamauchi for helpful discussion.

Correspondence should be addressed to Yu-shin Sou at ysodaka@juntendo.ac.jp or Masato Koike at mkoike@juntendo.ac.jp

https://doi.org/10.1523/ENEURO.0427-18.2019

Copyright (C) 2019 Sou et al.

This is an open-access article distributed under the terms of the Creative Commons Attribution 4.0 International license, which permits unrestricted use, distribution and reproduction in any medium provided that the original work is properly attributed.
We previously identified an anion channel that is localized to the Golgi apparatus and is a Golgi $\mathrm{pH}$ regulator (GPHR). The GPHR is essential for maintaining Golgi luminal acidification (Maeda et al., 2008). Using keratinocyte-specific GPHR knock-out mice, the GPHR was found to be essential for proper skin barrier function (Tarutani et al., 2012). Here, we took advantage of the GPHR conditional knock-out mice to better understand the morphologic and functional abnormalities in cerebellar circuits. We show that deletion of GPHR in cerebellar inhibitory circuits [PCs and basket cells (BCs)] leads to cerebellar ataxia due to PC degeneration. Impaired luminal acidification of the Golgi apparatus leads to vesiculation and fragmentation in PCs. Importantly, we found that axonal and synaptic abnormalities precede the loss of PCs. Moreover, the loss of GPHR in BCs causes disruption of inhibitory input to PC soma and further leads to climbing fiber (CF) innervation onto the soma of mutant PCs. These results indicate that GPHR-dependent Golgi luminal acidification plays an essential role in the structural and functional maintenance of PCs and BCs.

\section{Materials and Methods}

\section{Animals}

Male GPHR ${ }^{F / F}$ mice (Tarutani et al., 2012) and female GluD2-Cre knock-in mice (Yamasaki et al., 2011) were crossed to produce $G P H R^{F / F} ; G / u D 2-C r e$ mice. Male $G P H R^{F / F}$ mice and female L7/pcp2-Cre knock-in mice (Saito et al., 2005) were crossed to produce $G P H R^{F / F} ; L 7-$ Cre mice. In the present study, GPHR ${ }^{F /+}$ mice expressing Cre recombinase were used as the control. The procedures involving animal care, surgery and sample preparation were approved by the Animal Experimental Committee of Juntendo University and performed in accordance with the guidelines for the Care and Use of Laboratory Animals. Mice were housed in specific pathogen-free conditions with 12/12 h light/dark cycles at Juntendo University.

\section{Antibodies}

Preparation of rabbit anti-GPHR and anti-mGluR1 $\alpha$ antibodies were described previously (Maeda et al., 2008; Kamikubo et al., 2013). Goat anti-calbindin [(Af1040), RRID: AB_2571569], guinea pig anti-calbindin [(Af-280), RRID: AB_2571570], anti-parvalbumin [(Af-1000), RRID: $\left.A B \_2571615\right]$, rabbit anti-GABA $A_{A}$ receptor $\alpha 1$ subunit [GABA ${ }_{A} R \alpha 1$; (Af-660), RRID: AB_2571571], anti-vesicular GABA transporter [VGAT; (Af-500), RRID: AB_2571622], anti-type-1 vesicular glutamate transporter [vGlut1; (Af280), RRID: AB_2571616], and anti-vGlut2 [(Af-720), RRID: AB_2571619] were purchased from the Frontier Institute. Mouse anti-NeuN [(A60), RRID: AB_2298772] was purchased from Millipore. Rat anti-Mac 2 [(M3/38), RRID: AB_10060357] was purchased from Cedarlane. Rabbit anti-lbal [(019-19741), RRID: AB_839504] was purchased from Wako Pure Chemical Industries. Mouse antiGM130 [(35/GM130), RRID: AB_398141] was purchased from BD Biosciences. Sheep anti-TGN38 [(AAHP499), RRID: $A B$ 2287346] was purchased from AbD Serotec. A rat monoclonal antibody against GPHR was raised in rat using 
the peptide Cys-AHKQAPEKHMAP as the antigen. Rat hybridoma cells (Clone 35C5) were obtained by standard approaches.

\section{Plasmids}

The plasmid containing the open reading frame of the $\mathrm{pH}$ sensor protein, pHluorin, was kindly provided by Dr. James Edward Rothman (Miesenböck et al., 1998). We used the N-terminal 81 amino acids of human beta 1,4galactosyltransferase as a targeting signal for the Golgi apparatus. The plasmids, pHluorin-Golgi and pmKate2Golgi, were constructed based on pEYFP-Golgi (TAKARA/Clontech, catalog \#6909-1) by replacing the sequence encoding EYFP with the sequences encoding pHluorin and mKate2, respectively (Tanida et al., 2014). The DNA fragment encoding pHluorin-Golgi was introduced into the Nhel-EcoRI sites of pIRES, and the DNA fragment encoding mKate2-Golgi was introduced into the Sall-Notl sites of pIRES (TAKARA/Clontech, catalog \#Z1605N). The resultant plasmid was named as pHluorinGolgi-IRES-mKate2-Golgi.

\section{Primary culture of cortical neurons and adenovirus infection}

Primary cortical neurons were prepared according to a general protocol. Briefly, the cortex was dissected and dissociated from E14 mice embryos. Dissociated cortical cells were plated on poly-L-Ornithine (Sigma-Aldrich) coated 14-mm coverslips. Neurobasal medium (Thermo Fisher Scientific) supplemented with 2\% B27 (Thermo Fisher Scientific) and $2 \mathrm{mmol}$ L-glutamine (Nacalai Tesque) was used as the culture medium. After $2 \mathrm{~d}, 10$ $\mathrm{mmol} / \mathrm{L}$ cytosine arabinoside (Nacalai Tesque) was added to inhibit non-neuronal growth. Two days after this step, with the aim of achieving equal infection, the culture medium containing the adenovirus with 10 multiplicity of infection (MOI) was added to the medium. The lysates from cultured neurons were subjected to immunoblotting to detect GPHR and actin. Adenovirus vectors: Ad-CMViCre (\#1045) and Ad-GFP (\#1060) were purchased from Vector Biolabs.

\section{Quantitative real-time PCR}

cDNA was synthesized from $1 \mu \mathrm{g}$ of total RNA using the Transcriptor First-Strand cDNA synthesis kit (Roche Applied Science). Quantitative PCR was performed using the SYBR Green Real-time PCR Master Mix (Toyobo) with a Thermal Cycler Dice Real Time System II (TAKARA BIO). The signal intensity was normalized to the reference $\beta$-actin signal. The following primer sequences were used: GPHR left, 5'-CATGGTGCCCTTCTACATTG-3'; GPHR right, 5'-TCCCAGCTTCCAGAAGAAGT-3'; $\beta$-actin left, $5^{\prime}$-GCAAGCAGGAGTACGATGAG-3'; $\beta$-actin right, 5'-TCCCAGCTTCCAGAAGAAGT-3'.

\section{Measurement of pHluorin intensity}

pHluorin-Golgi and mKate2-Golgi were expressed by adenovirus-mediated transfection of pHluorin-GolgiIRES-mKate2-Golgi in primary cultured neurons using Lipofectamine 2000 (Thermo Fisher Scientific). Seven days after transfection, live-cell images of primary cultured neurons expressing pHluroin-Golgi-IRES-mKate2-Golgi were obtained using a FV1000 confocal laser scanning microscope (Olympus). The intensities of pHluorin and mKate2 in the same ROI were measured by the histogram analysis plugin in ImageJ (Schneider et al., 2012). The mean of the intensity ratio (pHluorin/mKate2) was calculated and processed for statistical analysis.

\section{Histological examination}

Deeply anesthetized mice were fixed by cardiac perfusion with $4 \%$ paraformaldehyde in $0.1 \mathrm{M}$ phosphate buffer $(\mathrm{pH}$ 7.4). Tissues were embedded in paraffin or OCTcompound (Tissue-Tek, 4583; Sakura Fintek). Threemicron paraffin sections were stained with $0.1 \%$ cresyl violet for Nissl staining. Meyer's hematoxylin and eosin $(\mathrm{H}$ \& E) staining was performed on $20-\mu \mathrm{m}$-thick cryosections. For immunofluorescence, frozen sections were subjected to antigen retrieval by incubating in target retrieval solution (S1699, Dako) for $10 \mathrm{~min}$ at $105^{\circ} \mathrm{C}$ and blocked with TNB buffer (0.1 M Tris- $\mathrm{HCl}, \mathrm{pH} 7.5,0.15 \mathrm{M} \mathrm{NaCl}$, and $0.5 \%$ TSA blocking reagent; FP102; PerkinElmer) at room temperature for $1 \mathrm{~h}$. The sections were then incubated with a mixture of primary antibodies at $4{ }^{\circ} \mathrm{C}$ overnight, followed by incubation with fluorescently labeled secondary antibodies [Alexa Fluor 488 (Thermo Fisher Scientific) for Cy3 or Cy5 (Jackson ImmunoResearch)] containing DAPI at room temperature for $1 \mathrm{~h}$. Fluorescence images were obtained using a FV1000 confocal laser scanning microscope (Olympus).

\section{Stereological sectioning and quantification of PCs}

The total number of PCs in the mouse cerebellum was estimated by stereological quantification and optical disector counting methods, as described previously (Chintawar et al., 2009, 2012; Ip et al., 2017). Sagittal cerebellar cryosections from control and GPHR ${ }^{F / F}$;GluD2-Cre mice (three mice per group) were serially cut at $20-\mu \mathrm{m}$ thicknesses to cover the entire cerebellum. Systematic random sampling of every 20th section collected was performed for a total of 18 sections per mice. Sections were immunostained with the anti-calbindin antibody to reveal PC soma and their dendrite in the molecular layer. Calbindin immunostaining images were visualized by using a fluorescence microscope BZ-X710 with a 20× objective lens (Keyence). The optical disector method was applied to identify and count PC tops and thus to calculate average PC densities $(N v)$. The reference volume (Vref) from PCs and molecular layers was obtained using Cavalieri's principle. Finally, the total number of PCs (Npc) was estimated according to the formula: $N p c=V r e f \times N v$. The images were analyzed using ImageJ cell counter and Grid layout plugins (Schneider et al., 2012).

\section{Electron microscopy (EM)}

Mice were fixed by cardiac perfusion with $4 \%$ paraformaldehyde in $0.1 \mathrm{M}$ phosphate buffer $(\mathrm{pH}$ 7.4). Cerebellar sections were post fixed with $2 \%$ paraformaldehyde and $2 \%$ glutaraldehyde in $0.1 \mathrm{M}$ phosphate buffer $(\mathrm{pH} 7.4)$ overnight followed by post fixation with $1 \% \mathrm{OsO}_{4}$, dehydration with a graded series of ethanol and embedding in Epon812 (Oken Shoji). Ultrathin sections were cut with an 
ultramicrotome UC6 (Leica Microsystems), stained with uranyl acetate and lead citrate and examined with a transmission EM HT7700 (Hitachi). For volume EM, images were taken with a focused ion beam scanning EM (FIBSEM) Helios Nanolab 660 (FEl Company). Epon blocks were milled by a focused ion beam and a serial block-face for every $20 \mathrm{~nm}$ was imaged using a backscattered electron detector (MD detector) at an acceleration voltage of $2.0 \mathrm{kV}$ and a current of $0.4 \mathrm{nA}$. 3D reconstruction was performed using Amira software (FEI Company).

\section{Measurement of the cerebellar area, PC density, and microglia density}

Measurement of the cerebellar area was conducted as described previously (Xu et al., 2018). Sagittal cerebellar cryosections (20 $\mu \mathrm{m}$ thick) from control and $G P H R^{F / F}$; GluD2-Cre mice (three mice per group) were serially cut and every 10th section was collected for $\mathrm{H}$ \& E staining. Cerebellar size was measured from five sections corresponding to positions 102-104 of the mouse brain atlas (Franklin and Paxinos, 2007). Sections were visualized by using a fluorescence microscope BZ-X710 with a $4 \times$ objective lens. Images were stitched into a whole cerebellum by the BZ-X image analyzer (Keyence) and the area of the cerebellum was measured. The mean area was calculated and processed for statistical analysis. For quantification of $P C$ densities, cerebellar sections from control and GPHR ${ }^{F / F}$;GluD2-Cre mice (three mice per group) were immunostained with the anti-calbindin antibody. The number of calbindin-positive PCs from each cerebellum was counted from a total of five sections, corresponding to positions 102-104 of the mouse brain atlas (Franklin and Paxinos, 2007). The PC density was calculated as the total number of PC somata divided by the sum of the area of PC and molecular layers. The mean PC densities were calculated and processed for statistical analysis. To measure microglia density, sections containing cerebellar lobule IX from control and GPHR ${ }^{F / F}$; GluD2Cre mice (three mice per group) were immunostained with the anti-IBA1 antibody, and nuclei were stained with DAPI. The number of total IBA1-positive cells was counted on five sections. The microglia density was obtained by dividing the IBA1-positive cells, with clearly visible nuclei, by the total area of the lobule. The mean density was obtained from all the microglia analyzed and processed for statistical analysis. All images were analyzed using ImageJ.

\section{Morphometry of PCs}

To quantify immunofluorescence images, five sections containing cerebellar lobule IX from each mouse were immunostained and analyzed using ImageJ. Measurements were obtained from three mice in each group. To calculate the ratio of PCs in which the Golgi apparatus is abnormally distributed, the number of PCs with such abnormality was divided by the total number of PCs. The abnormal distribution of the Golgi apparatus in PCs was defined by perinuclear accumulation of GM130 and TGN38, as revealed by immunostaining. The mean ratio of PCs with abnormal distribution of the Golgi apparatus was calculated and processed for statistical analysis. Cal- bindin immunostaining images were analyzed by ImageJ to quantify axon swelling, which enabled measurement of the size and number of axon swellings in the immunostained sections. An axonal torpedo area exceeding 15 $\mu \mathrm{m}^{2}$ was defined as swelling axons. The number of swelling axons was divided by the area of granule cell layer or deep cerebellar nuclei (DCNs) to calculate the density of axonal swelling. To count Pinceau structures, sections were immunostained with anti-calbindin and antiparvalbumin antibodies to elucidate BC axon terminals. The ratio of PCs with Pinceau structures was calculated as the total number of PCs with Pinceau structures divided by the total number of PCs. The ratio obtained was processed for statistical analysis. To quantify the frequency of mGluR $1 \alpha$ and vGlut2 single-positive or doublepositive puncta, analysis was restricted to the bottom two-thirds of the PC soma to avoid contamination of the analysis by puncta on dendrites. The ratio of vGlut2 puncta on PC soma was calculated by dividing the positive cells with the total number of cells analyzed. The mean obtained was processed for statistical analysis. For quantification of lamellar bodies, defined as smooth ERs with more than three stacks, in the cytoplasm of PCs, electron micrographs taken from control $(n=5, P 60)$ and GPHR ${ }^{F / F}$;GluD2-Cre $(n=5$, P60) mice were analyzed. The number of lamellar bodies was counted and divided by the total area of the PC cytoplasm to show lamellar body densities. The mean of lamellar body densities was calculated and processed for statistical analysis.

\section{Electrophysiology}

Electrophysiological analysis was performed by a conventional approach (Nakayama et al., 2012). Briefly, mice were decapitated following $\mathrm{CO}_{2}$ anesthesia, and the brains were rapidly removed and placed in chilled modified external solution $\left(0^{\circ} \mathrm{C}-4{ }^{\circ} \mathrm{C}\right)$ containing: $120 \mathrm{mM}$ choline-Cl, $2 \mathrm{mM} \mathrm{KCl}, 8 \mathrm{mM} \mathrm{MgCl}_{2}, 28 \mathrm{mM} \mathrm{NaHCO}_{3}, 1.25$ $\mathrm{mM} \mathrm{NaH} \mathrm{PO}_{4}$, and $20 \mathrm{mM}$ glucose, with bubbling of $95 \%$ $\mathrm{O}_{2}$ and $5 \% \mathrm{CO}_{2}, \mathrm{pH}$ 7.4. Parasagittal cerebellar slices (250 $\mu \mathrm{m}$ in thickness) were prepared by using a vibratome slicer (VT-1200S, Leica). For recovery, slices were incubated for at least $1 \mathrm{~h}$ in normal artificial CSF (ACSF) composed of $125 \mathrm{mM} \mathrm{NaCl}, 2.5 \mathrm{mM} \mathrm{KCl}, 2 \mathrm{mM} \mathrm{CaCl}_{2}, 1$ $\mathrm{mM} \mathrm{MgSO}_{4}, 1.25 \mathrm{mM} \mathrm{NaH}_{2} \mathrm{PO}_{4}, 26 \mathrm{mM} \mathrm{NaHCO}_{3}$, and 20 $\mathrm{mM}$ glucose, $\mathrm{pH} 7.4$, which was bubbled continuously with a mixture of $95 \% \mathrm{O}_{2}$ and $5 \% \mathrm{CO}_{2}$ at room temperature. Whole-cell recordings were made from visually identified PCs using an upright microscope (BX51WI, Olympus) at $32{ }^{\circ} \mathrm{C}$. The whole-cell pipette solution contained $140 \mathrm{mM} \mathrm{CsCl}, 10 \mathrm{mM}$ 2-[4-(2-hydroxyethyl)-1piperazinyl]ethanesulfonic acid (HEPES), $1 \mathrm{mM}$ ethylene glycol-bis(2-aminoethylether)-N, N, N', N'-tetraacetic acid (EGTA), $4.6 \mathrm{mM} \mathrm{MgCl}_{2}, 0.1 \mathrm{mM} \mathrm{CaCl}$, $4 \mathrm{mM} \mathrm{Na}-A T P$, and $0.4 \mathrm{mM}$ Na-GTP, pH 7.3, adjusted with $50 \% \mathrm{CsOH}, 287$ mOsm. Resistance of the patch pipette was 3-5 M $\Omega$. When $\mathrm{GABA}_{\mathrm{A}}$ receptor-mediated miniature IPSCs (mIPSCs) were recorded, tetrodotoxin (TTX; $1 \mu \mathrm{M}$ ) and 16-cyano-7-nitroquinoxaline-2,3-dione (CNQX; $10 \mu \mathrm{M}$ ) were present to block action potentials and AMPA receptor-mediated currents. An Axopatch 1D amplifier (Molecular Devices) was used and the 
Table1. Summary of statistical analyses

Figure, statistical test, and measure Fig. $1 B$

Quantitative real-time PCR

unpaired Student's $t$ test

Relative mRNA levels

Fig. $1 D$

Intensity of pHluorin

unpaired Student's $t$ test

Raito (pHluorin/mKate2; 488/562 nm)

Fig. $2 B$

Rotarod test

One-way ANOVA followed by

Sidak's multiple comoarusins test

Time (s)

Fig. $2 D$

Composite phenotype scoring system Kruskal-Wallis rank sum test followed by Dunn's test

Average score

Fig. 3B

Measurement of cerebellar area

One-way ANOVA followed by

Sidak's multiple comoarusins test

Area $\left(\mathrm{mm}^{2}\right)$

Fig. $3 E$

Quantitative analysis of PC density

One-way ANOVA followed by

Sidak's multiple comoarusins test

$\mathrm{PC} / \mu \mathrm{m}^{2}$

Related to Fig. 3F

Quantitative analysis of IBA1-positive

Microglia

Unpaired Student's $t$ test

Microglia/nm²

Related for Fig. $4 A$

Quantitative analysis of the abnormal

Golgi in PC

Unpaired Student's $t$ test

Ratio (abnormal Golgi distribution within

$\mathrm{PC} /$ total number of PCs)

Related to Fig. $5 A$

Quantitative analysis of axonal swelling

in granule cell layer

unpaired Student's $t$ test

Size of axon swelling $\left(\mu \mathrm{m}^{2}\right)$

Density of axon swellings $/ \mathrm{nm}^{2}$
$\mathrm{GPHR}^{F / F} \mathrm{AV}$ : GFP

mRNA levels of GPHR

$\begin{array}{ll}\text { Mean } & \text { SEM } \\ 0.992 & \pm 0.025\end{array}$

$G P H R^{F /+} \mathrm{AV}$ : Cre

Mean SEM

$1.015 \pm 0.038$

Age

$\mathrm{GPHR}^{\mathrm{Fl+}}$;GluD2-Cre

P45

P60

Mean

SEM

247.8

$\pm 15.39$

$231.0 \pm 23.67$

P90

$226.9 \pm 16.53$

$\mathrm{GPHR}^{\mathrm{Fl+}}$;GluD2-Cre

Age

P45

P60

P90

Mean

SEM

0

0

0.333

$\pm 0.167$

0.333

$\pm 0.167$

P150

0.222

$\pm 0.147$

P300

0.444

$\pm 0.176$

Age

$\mathrm{GPHR}^{\mathrm{F/+}}$;GluD2-Cre

P60

Mean

SEM

7.405

$\pm 0.0449$

7.340

$\pm 0.0569$

$\mathrm{GPHR}^{\mathrm{F/+}}$;GluD2-Cre

Age

P45

Mean

SEM

$\mathrm{P} 60$

0.0150

$\pm 0.00068$

0.0147

$+0.00093$

0.0158

$\pm 0.00117$

Age

GPHR ${ }^{F /+}$;GluD2-Cre

Mean

SEM

$35.69 \pm 2.70$

Age

GPHR ${ }^{\text {F/F }}$;GluD2-Cre

P45

Mean

SEM

66.85

$\pm 3.13$

$90.58 \pm 2.23$

15

15

\section{GPHR $^{\text {F/F }}$;GluD2-Cre}

Age

P45

Mean

SEM

$40.04 \pm 3.00$

$70.22 \pm 4.64$

GPHR $^{\text {F/F }}$;GluD2-Cre

Age

Mean

SEM

$56.54 \pm 4.94$

$\pm 8.59$

(Continued)
GPHR ${ }^{F / F} \mathrm{AV}$ : Cre mRNA levels of GPHR

Mean SEM $n$ Statistics

$0.044 \pm 0.011 \quad 6 \quad p<0.0001$

GPHR ${ }^{F / F} \mathrm{AV}$ : Cre

Mean SEM

$1.390 \pm 0.068$

$\rho=0.0013$

GPHR $^{\text {F/F }}$;GluD2-Cre

Mean SEM

$242.7 \pm 16.46$

$207.5 \pm 16.56$

$146.4 \pm 12.10$

Statistics

$p=0.9956$

$p=0.7158$

$p=0.0086$

GPHR ${ }^{F / F}$;GluD2-Cre

Mean SEM

$0.222 \quad \pm 0.147$

0.667

$\pm 0.167$

3.78

$\pm 0.222$

5.667

$\pm 0.236$

6.333

$\pm 0.441$

Statistics

$p>0.9999$

$p>0.9999$

$p=0.0087$

$p<0.0001$

$p=0.0002$

$\mathrm{GPHR}^{\mathrm{F} / \mathrm{F}}$;GluD2-Cre

Mean SEM

SEM
\pm 0.0816

n Statistics

$15 \quad 6.883$

$\pm 0.0974$

$15 p=0.0009$

6.127

15

$p<0.0001$

GPHR ${ }^{F / F}$;GluD2-Cre

$n$
15
15
15

Mean

SEM

0.01467

$\pm 0.00052$

$\begin{array}{ll}0.01261 & \pm 0.00049 \\ 0.00562 & \pm 0.00120\end{array}$

$n$
15

Statistics

0.00562

15

$p=0.9213$

$p=0.0002$

GPHR $^{\text {F/F }}$;GluD2-Cre

Mean SEM

$97.730 \pm 7.86$

$n$ Statistics

$15 p<0.0001$

Statistics

P45 vs P60

$p<0.0001$

Statistics

P45 vs P60

$p=0.0241$

Statistics

P45 vs P60

$p<0.0001$ 
Table1. Continued

Figure, statistical test, and measure

Related to Fig. 5C

Quantitative analysis of axonal swelling in DCNs

Unpaired Student's $t$ test

Density of axon swellings $/ \mathrm{nm}^{2}$

Related to Fig. $6 A$

Pinceau structure counting

One-way ANOVA followed by

Sidak's multiple comoarusins test

Raito (total number of PC with

Pinceau structure/total number of PCs)

Fig. $7 C, D$

Electrophysiological analysis

Unpaired Student's $t$ test

Frequency $(\mathrm{Hz})$

Amplitude (pA)

Related to Fig. $8 A$

vGlut2 positive puncta on PC soma

One-way ANOVA followed by

Sidak's multiple comoarusins test

Raito (vGlut2-positive puncta with PC

somata/total number of PCs)

Related to Fig. $8 B$

mGluR1 $\alpha$-vGlut2 double-positive puncta on PC soma

Unpaired Student's $t$ test

Raito (vGlut2-positive puncta with PC

somata/total number of PCs)

Related to Fig. 9

Quantitative analysis of

lamellar body density

Unpaired Student's $t$ test

Lamellar body/nm

\begin{tabular}{llcl}
\multicolumn{4}{l}{ GPHR } \\
Age & Mean & SIUD2-Cre & \\
P45 & 261.10 & \pm 32.54 & $n$ \\
P60 & 880.60 & \pm 46.07 & 15
\end{tabular}

Statistics

P45 vs P60

$p<0.0001$

$\begin{array}{llc} & \text { GPHR }^{F /+} \text {;GluD2-Cre } \\ \text { Age } & \text { Mean } & \text { SEM } \\ \text { P60 } & 89.58 & \pm 1.591\end{array}$

$\begin{array}{llc} & \text { GPHR }^{\text {F/F }} \text {;GluD2-Cre } \\ n & \text { Mean } & \text { SEM } \\ 15 & 17.92 & \pm 1.50\end{array}$

$n$ Statistics

GPHR ${ }^{F /+} ;$ GluD2-Cre

Age

Mean SEM

P60

$89.58 \quad \pm 1.591$

$\mathrm{GPHR}^{\mathrm{FF}+}$;GluD2-Cre

Age

Mean SEM

P60

17.92

$\pm 1.50$
$\mathrm{GPHR}^{\text {F/F }} ; \mathrm{L7}$-Cre
$n$ Mean SEM
15
87.46
$\pm 1.50$
GPHR $^{\text {F/F }}$ :L7-Cre
$n$ Mean SEM
15
87.46
$\pm 1.50$

$15 p<0.0001$

$n \quad$ Statistics

$15 p=0.7015$

$n \quad$ Statistics

$15 p<0.0001$

\begin{tabular}{lll} 
& \multicolumn{2}{l}{ GPHR } \\
$n$ & Mean & GluD2-Cre \\
4 & 0.892 & SEM \\
4 & 0.727 & \pm 0.2242 \\
4 & 0.165 & \pm 0.1690 \\
$n$ & Mean & SEM \\
4 & 73.15 & \pm 7.81 \\
4 & 61.16 & \pm 3.99 \\
4 & 138.90 & \pm 6.17
\end{tabular}

$n \quad$ Statistics

$4 \quad p=0.1258$

$\mathrm{GPHR}^{F /+}$;GluD2-Cre

All $\quad 1.340 \quad \pm 0.1135$

Small $0.9030 \quad \pm 0.0460$

Large $\quad 0.4360 \pm 0.0833$

Mean SEM

All $\quad 99.82 \quad \pm 3.57$

Small $70.00 \quad \pm 4.97$

Large $\quad 162.60 \quad \pm 19.87$

$\begin{array}{llc} & \text { GPHR } & \text { F/+ } ; \text { GluD2-Cre } \\ \text { Age } & \text { Mean } & \text { SEM } \\ \text { P60 } & 9.95 & \pm 1.07\end{array}$

$\begin{array}{lll} & \text { GPHR }^{F / F} ; \text { GluD2-Cre } \\ n & \text { Mean } & \text { SEM } \\ 15 & 69.02 & \pm 4.21\end{array}$

n Statistics

$15 p<0.0001$

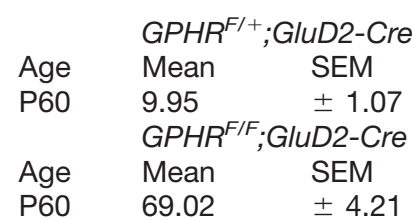

$\begin{array}{lll} & \text { GPHR }^{\text {F/F }} ; L 7-C r e \\ n & \text { Mean } & \text { SEM } \\ 15 & 15.06 & +1.300\end{array}$

$15 \quad 15.06 \pm 1.30$

GPHR $^{\text {F/F }}$;L7-Cre

$n$ Mean SEM

$15 \quad 15.06 \quad \pm 1.300$

n Statistics

$15 p=0.4383$

n Statistics

$15 p<0.0001$

$\begin{array}{lll} & \text { GPHR } & \text { F/+ } \\ \text { AgluD2-Cre } & \text { Mean } & \text { SEM } \\ \text { P60 } & 9.34 & \pm 1.26\end{array}$

$15 p<0.0001$ n Statistics signal was filtered at $5 \mathrm{kHz}$, digitized at $10 \mathrm{kHz}$ and stored on a personal computer (pClamp 10, Molecular Devices).

\section{Behavioral tests}

The accelerating rotarod test was performed on a rotarod machine with automatic timers and falling sensors (MK-660D, Muromachi Kikai). Control and GPHR ${ }^{F / F}$; GluD2-Cre mice ( $n=5$ for each genotype of females) were placed on a rotating rod, rotation was accelerated to $40 \mathrm{rpm}$ for $300 \mathrm{~s}$, and the latency period for each mouse to fall off was measured. If a mouse remained on the rod for $>300 \mathrm{~s}$, the latency period was recorded as $300 \mathrm{~s}$. Mice underwent three trials per day and the mean latency period of the three trials was considered for statistical analysis. Cerebellar ataxic phenotypes of control and GPHR ${ }^{F / F}$;GluD2-Cre mice (three mice per group) were evaluated at P45, P60, P90, P150, and P300 using the composite phenotype scoring system based on hindlimb clasping, open field gait and ledge tests (Guyenet et al., 2010). Each test was repeated three times. All tests were 
scored on a scale of $0-3$, with a combined total of $0-9$ for all three tests. For the limb clasping test, mice were lifted for $20 \mathrm{~s}$ by grasping their tail and movement of the hind limbs was scored as follows. A score of 0 indicates a mouse spreading their hind limbs away from the abdomen. A score of 1 indicates a mouse pulling their hindlimb partially toward their abdomen for $>5 \mathrm{~s}$. A score of 2 indicates a mouse pulling both hind limbs partially toward their abdomen for $>5 \mathrm{~s}$. A score of 3 indicates a mouse retracting hind limbs and touching the abdomen for $>50 \%$ of the observation time. For the open field gait test, mice were placed on a flat surface and oriented away from the researcher. A score was recorded as follows. A score of 0 indicates that a mouse moved normally and its abdomen did not touch the surface. A score of 1 indicates that tremor was observed or the mouse appeared to limp while walking. A score of 2 indicates a mouse showing severe tremor, severe limp, lowered pelvis or feet pointing away from the body. A score of 3 indicates that a mouse had difficulty to move forward and dragged their abdomen along the surface. For the ledge test, mice were lifted from a cage and placed on the ledge of the cage. A score was recorded as follows. A score of 0 indicates that a mouse walked along the ledge without losing its balance and went back into the cage using its paws. A score of 1 indicates that a mouse lost its footing while walking on the ledge. A score of 2 indicates that a mouse was unable to walk effectively on or let itself down from the ledge. A score of 3 indicates that a mouse was unable to walk, get down from the ledge, or simply fell off. All behavioral tests were performed by researchers blinded to the genotype of the mice.

\section{Statistical analysis}

See Table 1 for a summary of statistical results relating to this work. Actual $p$ values and the number of samples are stated in the text, figure legends, and Table 1. All statistical analyses were performed using GraphPad Prism 6 (GraphPad Software Inc.; RRID: SCR_002798). At least three independent samples were used for assessing statistical significance. Normality of the datasets was determined by the Kolmogorov-Smirnov test. Comparisons between multiple experimental groups for the accelerating Rotarod test, measurement of cerebellar area, quantitative analysis of PC density, Pinceau structure counting and quantification of the frequency of vGlut2 were made using one-way ANOVA followed by Sidak's multiple comparisons test. For comparing two experimental groups for quantitative real-time PCR, quantitative analysis of the pHluorin intensity, density of microglia, abnormal distribution of the Golgi apparatus, quantitative analysis of axonal swelling, electrophysiological analysis and quantitative analysis of the lamellar body density were conducted using the unpaired Student's $t$ test. For combined scores of the composite phenotype scoring system, which did not follow a normal distribution according to the Kolmogorov-Smirnov test, a non-parametric test was performed using the Kruskal-Wallis rank sum test followed by the Dunn's test. The data are presented as the mean \pm
SEM $; p<0.05$ was considered statistically significant and $p<0.01$ was considered highly significant.

\section{Results}

\section{Elevated luminal pH of the Golgi apparatus in primary cultured neurons lacking GPHR}

Initially, we assessed the disruption of the GPHR gene in primary cultured neuron from GPHR floxed embryos by using an adenovirus expression system. Deletion of the GPHR protein was confirmed by immunoblot analysis using an anti-GPHR antibody in primary cultured neurons from GPHR floxed mice expressing Cre recombinase (Fig. $1 A)$. As expected, exogenous expression of Cre recombinase suppressed GPHR mRNA levels in primary cultured neurons, as examined by quantitative real-time PCR (Fig. 1B; Table 1; GPHR ${ }^{F / F}$; AV: GFP: $0.992 \pm 0.025, n=$ 6; GPHR $R^{F / F}$; AV: Cre: $0.044 \pm 0.011, n=6$; unpaired Student's $t$ test, $\left.t_{(10)}=35.41, * * p<0.0001\right)$. The function of GPHR for maintaining the luminal pH of the Golgi apparatus in neurons was examined by constructing a signal localized at the Golgi apparatus to the $\mathrm{pH}$ sensor protein pHluorin and red fluorescence protein mKate2, respectively (Miesenböck et al., 1998; Tanida et al., 2014). Using the constructed tandem expression plasmid (i.e., pHluorin-Golgi-IRES-mKate2-Golgi), alkalization of the Golgi apparatus lumen in response to deletion of GPHR in primary cultured neurons was monitored. The fluorescence intensity of pHulorin markedly increased, indicating that deletion of GPHR elevated the luminal pH of the Golgi apparatus in primary cultured neurons (Fig. 1C). Quantitative measurements confirmed that the pHluorin/mKate2 fluorescent ratio of primary cultured neurons lacking GPHR (GPHR $R^{F / F}$; AV: Cre) is higher than that of the control $\left(G P H R^{F /+}\right.$; AV: Cre) neurons (Fig. 1D; Table 1; GPHR $R^{F /+}$; AV: Cre: $1.015 \pm 0.038, n=5$; GPHR ${ }^{F / F}$; AV: Cre: $1.390 \pm$ $0.068, n=5$; unpaired Student's $t$ test, $t_{(8)}=4.827, * * p=$ $0.0013)$. These results suggest that GPHR is responsible for maintaining normal luminal acidic conditions of the Golgi apparatus in neurons.

\section{GPHR ${ }^{F / F}$;GluD2-Cre mice show cerebellar ataxia with progressive neurodegeneration}

Next, to study the function of GPHR in neurons in vivo, we generated PC- and BC-specific GPHR knock-out mice by crossing GPHR floxed mice with GluD2-Cre mice, in which Cre recombinase was knocked-in into the glutamate receptor $\delta 2$ (GluD2) locus (Yamasaki et al., 2011). Cre-mediated deletion of GPHR in PCs was completed by postnatal day (P) 45 (Fig. 2A). Results from the rotarod test showed that GPHR ${ }^{F / F}$; GluD2-Cre mice showed uncoordinated movement from P90 (Fig. 2B; Table 1; oneway ANOVA, $n=5$ mice for each group, female; $F_{(5,24)}=$ 4.753, $p=0.0037$; and Sidak's post hoc test, control vs GPHR ${ }^{\text {F/F }}$;GluD2-Cre, $p=0.9956$ at P45, $p=0.7158$ at $\mathrm{P} 60$ and $* * p=0.0086$ at P90). Composite phenotype scoring of the hindlimb clasping, open field gait and ledge tests (Guyenet et al., 2010) confirmed that GPHRF/F; GluD2-Cre mice older than P90 displayed ataxic phenotypes as judged by loss of gait coordination and balance, reduced locomotor activity and abnormal hindlimb clasp- 

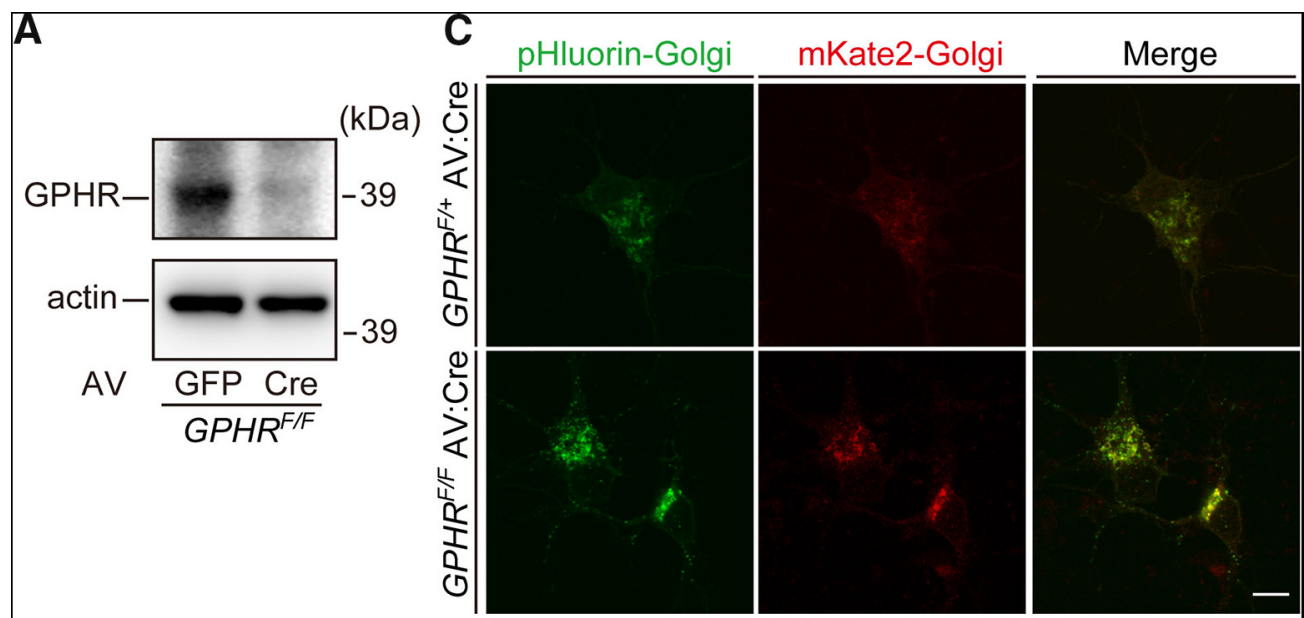

B
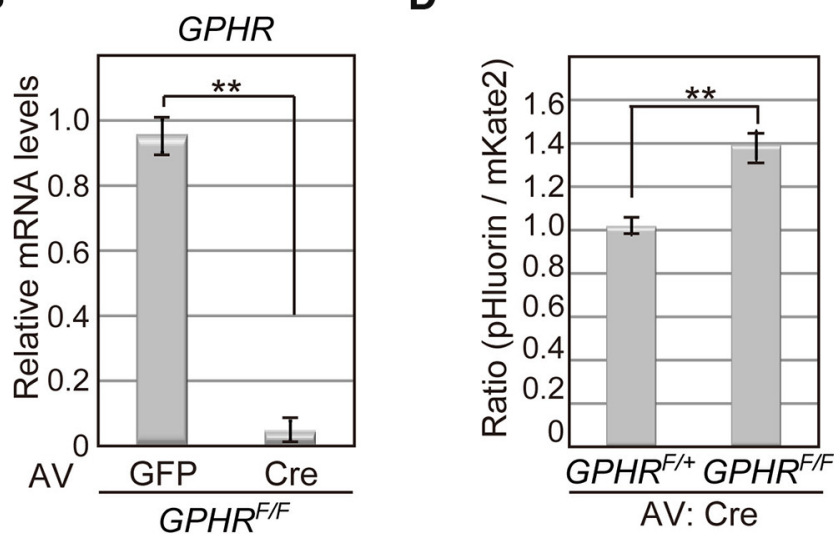

Figure 1. Altered luminal $\mathrm{pH}$ of the Golgi apparatus in GPHR deficient primary cultured neurons. $\boldsymbol{A}$, Immunoblot analysis in primary cultured neurons from GPHR ${ }^{F / F}$ mice with adenovirus (AV)-based exogenous expression of GFP or Cre recombinase. Nine days after infection, total cell lysates were subjected to immunoblot analysis for GPHR and actin. Data are representative of three independent experiments. B, Quantitation of mRNA levels of GPHR in primary cultured neurons manipulated, as described in $\boldsymbol{A}$. Data are presented as the mean $\pm \mathrm{SEM} ; n=6$ for each group; $* * p<0.01$ (unpaired Student's $t$ test). $\boldsymbol{C}$, Fluorescence images of pHluorin-Golgi in primary cultured neurons from GPHR ${ }^{F /+}$ and GPHR ${ }^{F / F}$ mice with AV-based exogenous expression of Cre recombinase. Two days after infection, pHluorin-Golgi-IRES-mKate2-Golgi was transfected. Fluorescence images were taken seven days after transfection. Data are representative of three independent experiments. Scale bar: $20 \mu \mathrm{m}$. D. Quantification of the pHluorin/mKate2 fluorescence ratio in primary cultured neurons manipulated as described in $\boldsymbol{C}$. Data are presented as the mean $\pm \mathrm{SEM} ; n=5$ for each group; $* * p<$ 0.01 (unpaired Student's $t$ test).

ing, which are commonly observed in mice with cerebellar degeneration (Fig. 2C,D; Movie 1; Table 1; Kruskal-Wallis test, three mice per group; three trials per mouse; $n=9$ trials for each group, KS $=69.33, p<0.0001$; Dunn's post hoc test, control vs GPHR ${ }^{F / F} ;$ GluD2-Cre, $p>0.9999$ at $\mathrm{P} 45, p>0.9999$ at $\mathrm{P} 60, * * p=0.0087$ at $\mathrm{P} 90, * * p<$ 0.0001 at $\mathrm{P} 150, * * p=0.0002$ at $\mathrm{P} 300$ ). These phenotypes indicated that deletion of GPHR in PCs and BCs leads to cerebellar ataxia.

$H$ \& $E$ staining at $P 60$ revealed difference in the size of the cerebellum between control and GPHR ${ }^{F / F}$;GluD2-Cre mice, and at P90 the difference was more pronounced (Fig. $3 A, B$; Table 1; one-way ANOVA, three mice per group; five sections per mouse; $n=15$ section for each group, $F_{(3,56)}=42.26, p<0.0001$; and Sidak's post hoc test, control vs GPHR ${ }^{F / F} ;$ GluD2-Cre, $* * p=0.003$ at P60, control vs GPHRF/F;GluD2-Cre, **p $<0.0001$ at P90). The histological feature of PC lost was detected in $G P H R^{F / F}$;
GluD2-Cre mice at P60 and was more severe at P90, as examined by Nissl staining (Fig. $3 C$ ). Consistent with Nissl staining, loss of PCs was also detected by calbindin immunostaining. GPHR ${ }^{F / F}$;GluD2-Cre mice exhibited loss of PCs especially in lobule IX at P60 and a decrease in PC density was observed in whole cerebellar lobules at P90, as examined by calbindin immunostaining (Fig. 3D,E; Table 1; one-way ANOVA, three mice per group; five sections per mouse; $n=15$ sections for each group, $F_{(5,96)}=112.2, p<0.0001$; and Sidak's post hoc test, control vs GPHR ${ }^{F / F}$;GluD2-Cre, $p=0.9213$ at P45, **p = 0.002 at $\mathrm{P} 60, * * p<0.0001$ at $\mathrm{P} 90)$. The number of PCs in control and $G P H R^{F / F}$;GluD2-Cre mice by stereological methods were estimated to confirm these histological results (Table 2). Comparison of the number of PCs in the cerebellum between control and GPHR ${ }^{F / F}$;GluD2-Cre mice at P90 revealed that there were only $\sim 20 \%$ of the $\mathrm{PC}$ remaining in GPHR ${ }^{F / F}$;GluD2-Cre mice. These results 


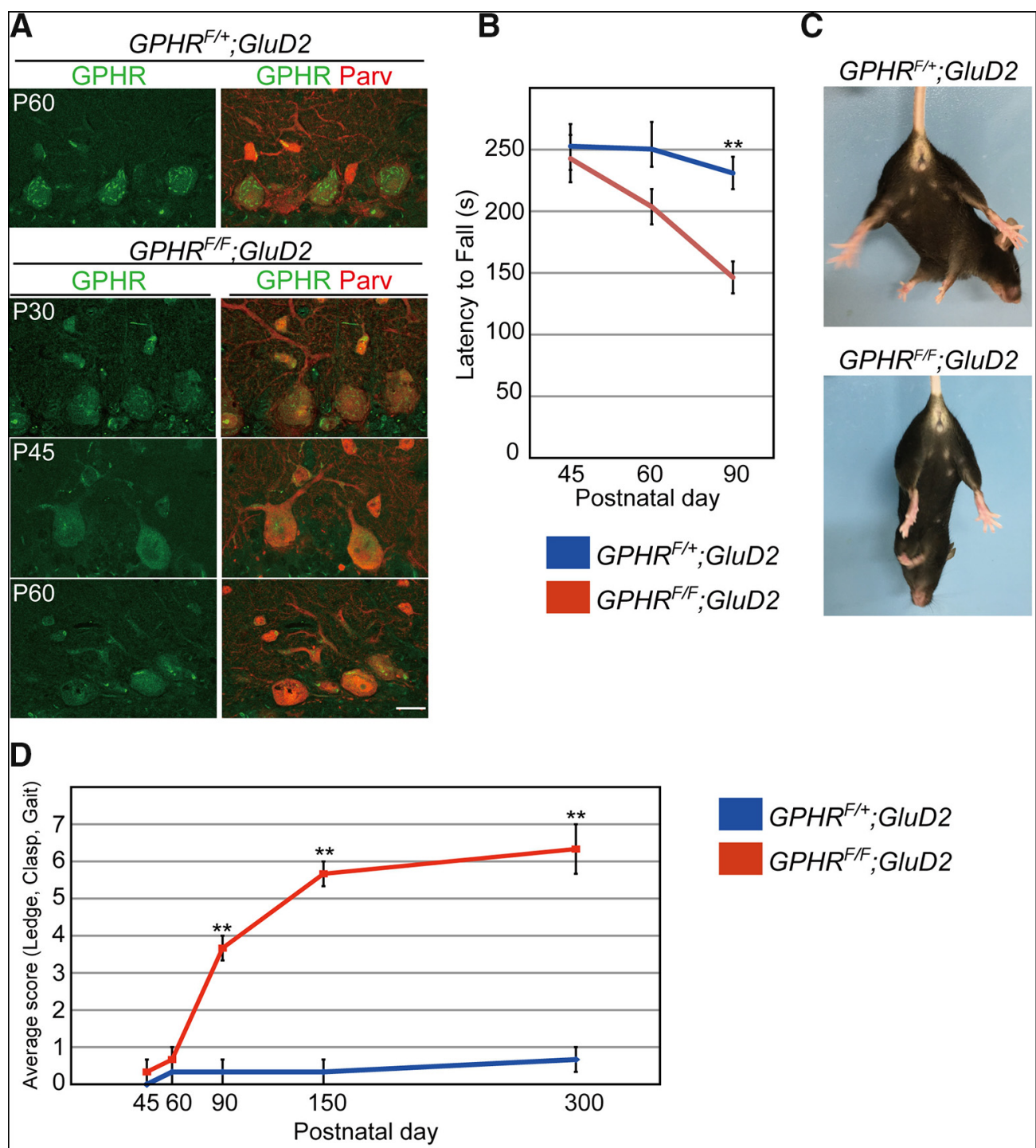

Figure 2. GPHR ${ }^{F / F}$;GluD2-Cre mice lack motor coordination. A, GPHR is deleted in PCs. Double immunofluorescence for GPHR (green) and parvalbumin (Parv; red) in the PC layer from GPHR ${ }^{F /+}$;GluD2-Cre mice at P60 and GPHR ${ }^{F / F}$;GluD2-Cre mice at P30, P45, and P60. Dotted immunoreactivity for GPHR is not discernable in PCs from GPHR ${ }^{F / F}$;GluD2-Cre mice after P45. Scale bars: $20 \mu \mathrm{m}$. $B$, Accelerating rotarod test for $G P H R^{F /+}$;GluD2-Cre and $G P H R^{F / F} ;$ GluD2-Cre mice. The average retention time on the rotarod is shown ( $n=5$ mice, female); **p $<0.001$ (one-way ANOVA with Sidak's post hoc test). C, Limb clasping reflex in GPHR ${ }^{F / F}$;GluD2-Cre mice at P90. When lifted by the tail, GPHR ${ }^{F /+}$;GluD2-Cre mice extended their hind limbs, whereas GPHR ${ }^{F / F}$;GluD2-Cre mice moved their legs to the trunk. $D$, Behavioral analyses of $G P H R^{F / F} ; G / u D 2-C r e$ mice relative to $G P H R^{F /+}$;GluD2-Cre mice. Graph showing cerebellar ataxic phenotypes score of $\mathrm{GPHR}^{F /+}$;GluD2-Cre and GPHR ${ }^{F / F}$;GluD2-Cre mice at P45, P60, P90, P150, and P300 (three mice per group). Average composite score for each genotype at each age was calculated. Data are presented as the mean \pm SEM; $n=9$ trial for each group, $* * p<0.01$ (Kruskal-Wallis test followed by the Dunn's test). For $\boldsymbol{A}$, three mice per genotype; $n=5$ sections per mouse; representative images are shown. See also Movie 1.

suggest that the number of PCs in GPHR/F;GluD2-Cre mice decreases in an age-dependent manner. Corresponding to the degenerative changes in PCs, activated microglia immunopositive for IBA1 and/or MAC2 were abundant in the cerebellum of GPHR ${ }^{F / F}$;GluD2-Cre mice (Fig. 3F). Quantification confirmed that the density of IBA1immunopositive microglia in lobule IX increased significantly in $G P H R^{F / F}$;GluD2-Cre mice when compared with that of control mice (Table 1; GPHR ${ }^{F /+}$;GluD2-Cre: $35.69 \pm$ 2.706; GPHR ${ }^{F / F}$;GluD2-Cre: $97.73 \pm 7.861$, three mice per group; five sections per mouse; $n=15$ sections for each group, unpaired Student's $t$ test, $t_{(28)}=7.463, * * p<$ 0.0001).

\section{GPHR loss causes abnormal morphology of the Golgi apparatus}

Alkalization of the Golgi luminal pH causes morphological abnormalities of the Golgi apparatus in cultured cells (Tartakoff, 1983; Sakaguchi et al., 1996; Maeda et al., 2008). However, very little information can be gleaned from in vivo literature. In normal PCs, the Golgi apparatus is properly distributed by forming a perinuclear strand 


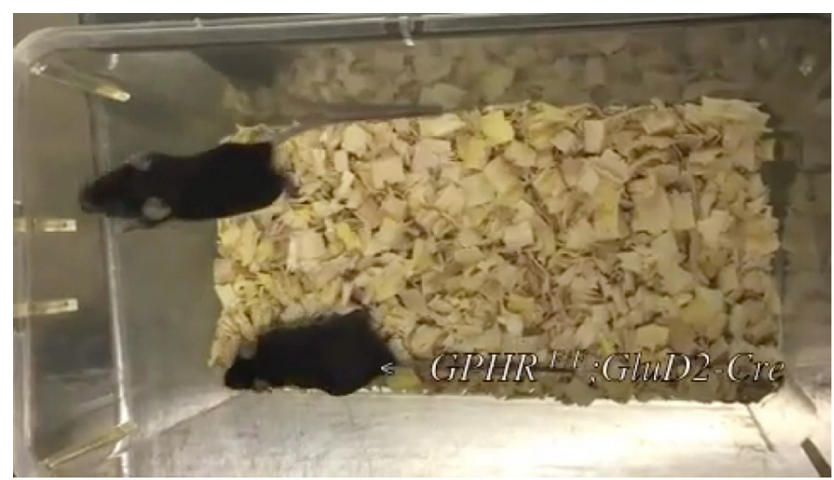

Movie 1. Lack of motor coordination in $G P H R^{F / F}$;GluD2-Cre mice. GPHR ${ }^{F / F}$;GluD2-Cre mice display abnormal gait at P90. The control littermates display a normal phenotype. [View online]

(Palay and Chan-Palay, 1974; Liu et al., 2017). To characterize the Golgi apparatus in PCs of $\mathrm{GPHR}^{F / F}$;GluD2Cre mice, we performed double staining for GM130 and TGN38, which are markers for cis- and trans-Golgi, respectively. In control PCs, GM130- and TGN38-positive Golgi signals were distributed as perinuclear small patches, which were enriched at the apical pole facing the primary dendrite. Each Golgi stack was designated as cisand trans-cisterns. In contrast, GPHR deficiency caused perinuclear accumulation of the immunopositive areas and some were shifted to the basolateral perikarya at P45, indicating an abnormal distribution of the Golgi apparatus (Fig. 4A). After P45, the Golgi apparatus appeared as a mixture of GM130- and TGN38-positive areas (Fig. 4A). Quantification confirmed that the ratio of the abnormal Golgi apparatus distribution in PCs of $G P H R^{F / F}$;GluD2Cre mice increased significantly in an age-dependent manner (Table 1; GPHR ${ }^{F / F}$;GluD2-Cre at P45: $66.85 \pm$ 3.13; GPHR $^{F / F}$;GluD2-Cre at P60: $90.58 \pm 2.23$, three mice per group; five sections per mouse; $n=15$ sections for each group; GPHR ${ }^{F / F}$;GluD2-Cre at P45: 185 PCs; GPHR ${ }^{F / F}$;GluD2-Cre at P60: 163 PCs; unpaired Student's $t$ test, $\left.t_{(28)}=6.174, * * p<0.0001\right)$. EM showed that instead of stacks of flattened cisterns, clusters of vacuolar structures were observed in GPHR-deficient PCs at P45, suggesting fragmentation of the Golgi apparatus (Fig. 4B). Furthermore, volumetric reconstruction of the Golgi apparatus by FIB-SEM confirmed fragmentation of the Golgi apparatus with clusters of vacuoles in PCs from GPHRF/F; GluD2-Cre mice (Fig. 4C; Movies 2, 3). These data indicated that acidification of the luminal $\mathrm{pH}$ of the Golgi apparatus is important for maintaining Golgi apparatus morphology and distribution in PCs.

\section{Golgi abnormality is accompanied by axonal and synaptic impairment in PCs}

We reported previously that the regulation of luminal $\mathrm{pH}$ of the Golgi apparatus is indispensable for ensuring the normal function of the Golgi apparatus, such as glycosylation and vesicular transport in cultured cells (Maeda et al., 2008). In addition, the Golgi apparatus is important for axonal transport of neurotransmitters in mature neurons (Campenot et al., 2003; Horton et al., 2005; Matsuki et al., 2010; Liu et al., 2017). Thus, morphological alterations in $\mathrm{PC}$ axons on perturbations in luminal $\mathrm{pH}$ of the Golgi apparatus were investigated. PC axons traverse the granule cell layer to innervate neurons in DCN. First, we focused on PC axons in the granule cell layer. Different from control axons at P60 (Fig. 5A), calbindin immunostaining revealed that axon swellings appeared at P45 in GPHR ${ }^{\text {FIF; }}$ GluD2-Cre mice, and these areas increased in size and number at P60 (Table 1; size: GPHR ${ }^{F / F}$;GluD2Cre at P45: $40.04 \pm 2.99 \mu \mathrm{m}^{2} ;$ GPHR $^{F / F}$;GluD2-Cre at P60: $70.22 \pm 4.63 \mu \mathrm{m}^{2}$, unpaired Student's $t$ test, $t_{(28)}=$ $6.405, * * p<0.0001$; density of GPHR ${ }^{F / F}$;GluD2-Cre mice at P45: $56.54 \pm 4.94 / \mathrm{nm}^{2} ;$ GPHR $^{F / F} ;$ GluD2-Cre at P60: $80.19 \pm 8.56 / \mathrm{nm}^{2}$, unpaired Student's $t$ test, $t_{(28)}=2.384$, $* p=0.0241$; three mice per group; five sections per mouse; $n=15$ sections for each group). EM observations revealed myelinated swelling axons in the granular cell layer at P45 in GPHR $R^{F / F}$;GluD2-Cre mice (Fig. $5 B$ ). In the $D C N$ region of GPHR ${ }^{F / F}$;GluD2-Cre mice, swelling axons also appeared at $\mathrm{P} 45$ and increased prominently in number at P60 (Fig. $5 C$; Table 1; density of GPHR ${ }^{F / F}$;GluD2Cre mice at P45: $261.1 \pm 32.54 / \mathrm{nm}^{2} ; \mathrm{GPHR}^{F / F}$;GluD2-Cre at P60: $880.6 \pm 46.07 / \mathrm{nm}^{2}$, three mice per group; five sections per mouse; $n=15$ sections for each group, unpaired Student's $t$ test, $t_{(28)}=10.98, * p<0.0001$ ). Many of these swelling axons were positive for calbindin but negative for the vGAT, which would reflect swelling axons containing materials other than synaptic vesicles, as shown in the EM observations (Fig. 5C,D). Interestingly, some presynaptic PC terminals exhibited an abnormal ultrastructural profile and showed symmetrical synaptic contacts with electron-dense postsynaptic density (Fig. $5 E$ ). In addition, we found that axosomatic synapses on individual DCN neurons were reduced as early as P45, leading to intercellular spaces being created around the DCN neurons (Fig. 5D-F). These results indicated that the impairment of acidic conditions in the Golgi apparatus causes axonal and synaptic abnormalities in PCs.

\section{GPHR is required for maintaining the Pinceau structure}

Next, we investigated the innervation of BCs, one of the GABAergic interneurons, to the somatodendritic compartment of PCs. The axons of BCs extend toward the bottom of the PC soma and their branches are densely terminated around the axon initial segment (AIS), forming a structure known as the Pinceau (Palay and Chan-Palay, 1974; Gianola et al., 2003; Ango et al., 2004; Sotelo, 2008). We performed double immunostaining of parvalbumin, which labels both PCs and GABAergic interneurons in the cerebellar molecular layer and calbindin that selectively labels PCs. While control BC axons completely surrounded the PC somata forming Pinceau structures around AIS at P60, GPHR ${ }^{F / F}$;GluD2-Cre mice exhibited markedly lower numbers of Pinceau structures at P60 (Fig. 6A). To confirm whether the loss of Pinceau structures is because of GPHR-deficiency in BCs, we further analyzed this phenomenon using the PC-specific GPHR knock-out mice, $G P H R^{F / F} ; L 7-C r e$ mice. Interestingly, the Pinceau structures remained intact in $G P H R^{F / F} ; L 7-C r e$ mice at $P 60$ and 


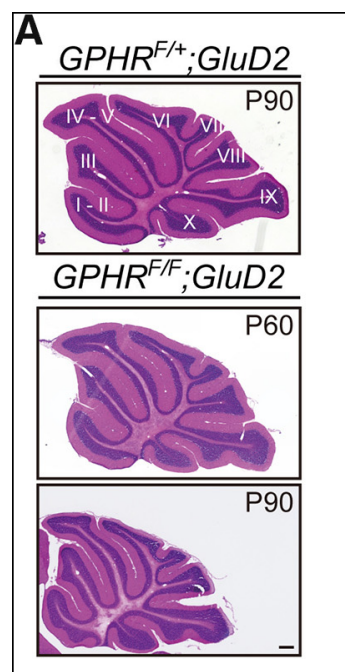

D
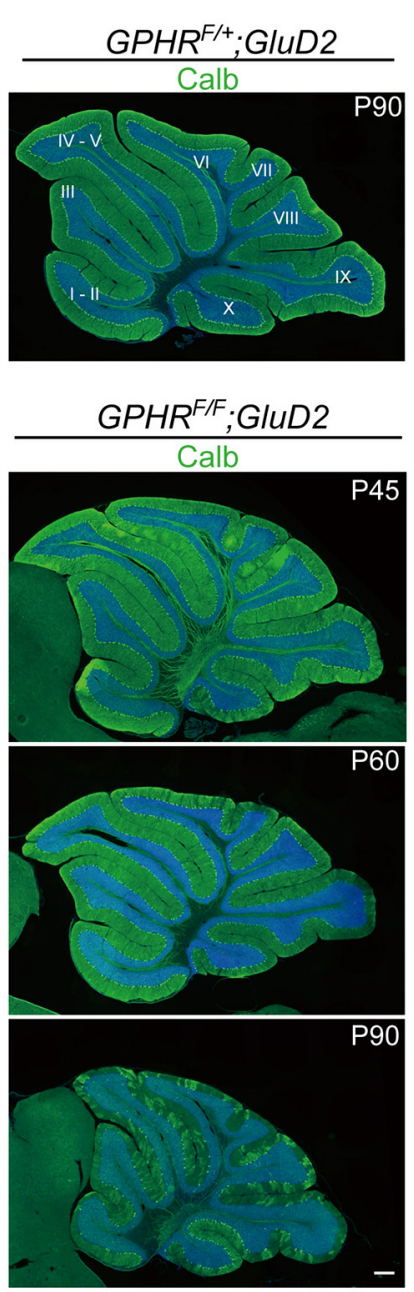
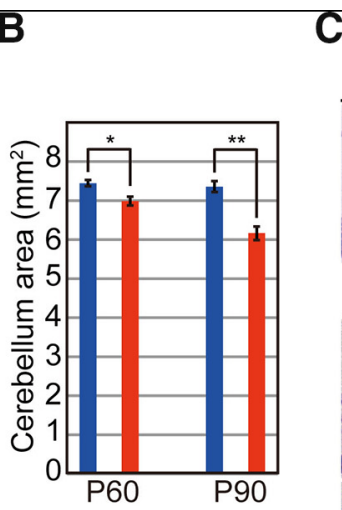

$\mathrm{GPHR}^{\mathrm{F/+}}$;GluD2 GPHR ${ }^{F / F}$;GluD2

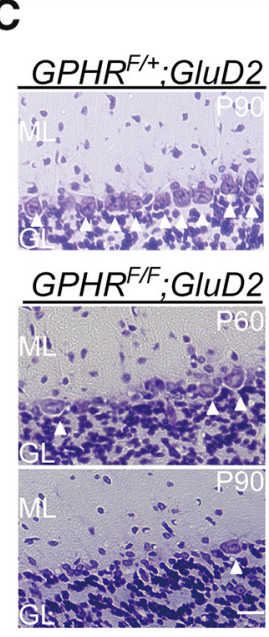

E

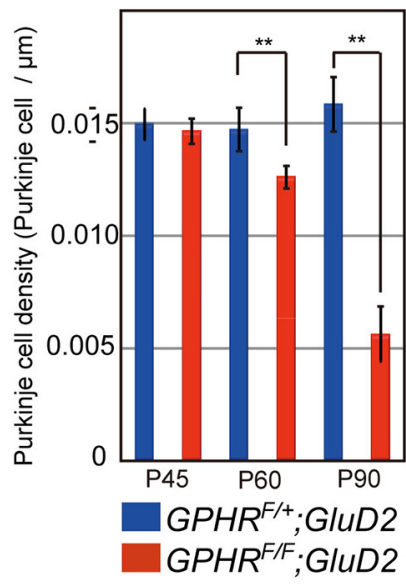

F
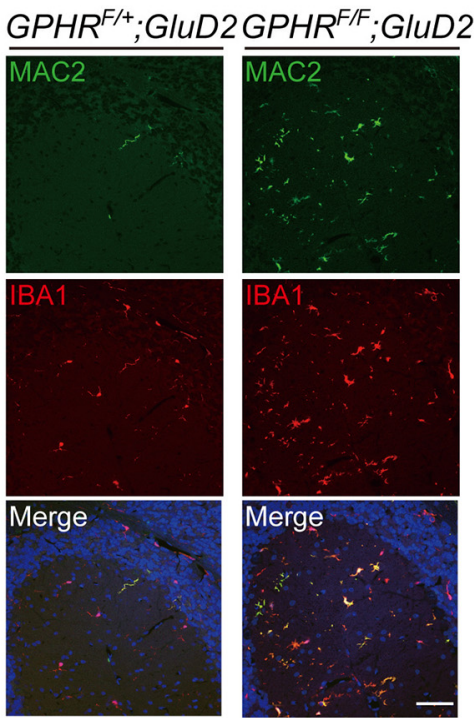

Figure 3. Progressive neurodegeneration in $G P H R^{F / F}$;GluD2-Cre mice. $A, H$ \& E-stained images of sagittal sections from $G P H R^{F /+}$; GluD2-Cre mice at P90 and GPHR ${ }^{F / F}$;GluD2-Cre mice at P60 and P90. Cerebellar lobules are indicated by Roman numerals. Scale bar: $300 \mu \mathrm{m}$. B, Measurement of cerebellar areas in GPHR ${ }^{F /+} ;$ GluD2-Cre and GPHR ${ }^{F / F}$;GluD2-Cre mice at P60 and P90 (three mice per group). Five sections from each mouse corresponding to the cerebellar region, as shown in $\boldsymbol{A}$, were measured. Data are presented as the mean \pm SEM; $n=15$ sections for each group, $* p<0.05, * * p<0.01$ (one-way ANOVA with Sidak's post hoc test). $\boldsymbol{C}$, Nissl staining of the PC layer of lobule IX in the cerebellum from GPHR ${ }^{F /+} ;$ GluD2-Cre mice at P90 and GPHR ${ }^{F / F}$;GluD2-Cre mice at P60 and P90. Arrowheads indicate PCs. ML, molecular layer; GL, granule cell layer. Scale bar: $30 \mu \mathrm{m}$. $\boldsymbol{D}$, Immunofluorescence for calbindin (Calb; green) in the cerebellum of $\mathrm{GPHR}^{F /+}$;GluD2-Cre mice at P90 and GPHR ${ }^{F / F}$;GluD2-Cre mice at P45, P60, and P90. Nuclei are 
continued

stained with DAPI (blue). Cerebellar lobules are indicated by Roman numerals. Scale bar: $300 \mu m$. $\boldsymbol{E}$, The density of PCs was measured following immunofluorescence staining for calbindin GPHR ${ }^{F /+} ; G / u D 2-C r e$ and $G P H R^{F / F}$;GluD2-Cre mice at P45, P60, and P90. Five sections from each mouse corresponding to the cerebellar region as shown in $\boldsymbol{D}$ were measured. Data are presented as the mean $\pm \mathrm{SEM} ; n=15$ sections for each group; $* * p<0.01$ (one-way ANOVA with Sidak's post hoc test). $\boldsymbol{F}$, Double immunofluorescence for IBA1 (green) and MAC2 (red) in the cerebellum of GPHR ${ }^{F /+}$;GluD2-Cre and GPHRF/F;GluD2-Cre mice at P60. Nuclei are stained with DAPI (blue). Scale bar: $50 \mu \mathrm{m}$. For $\boldsymbol{A}, \boldsymbol{C}, \boldsymbol{D}, \boldsymbol{F}$, three mice per genotype; $n=5$ sections per mouse; representative images are shown.

even after the loss of PCs (Fig. 6A, arrows). Quantification confirmed that the mean Pinceau frequency was significantly reduced in GPHR ${ }^{F / F}$;GluD2-Cre mice when compared with that of control and GPHR ${ }^{F / F} ;$ L7-Cre mice (Table 1; control: $89.58 \pm 1.59 \%$; GPHR ${ }^{F / F}$;GluD2-Cre: $17.92 \pm 1.50 \%$; GPHR ${ }^{F / F}$;L7-Cre: $87.46 \pm 1.50 \%$, three mice per group; five sections per mouse; $n=15$ sections for each group; GPHRF/+;GluD2-Cre: 203 PCs; GPHR ${ }^{F / F}$; GluD2-Cre: 156 PCs; GPHRF/F; L7-Cre: 160 PCs; oneway ANOVA, $F_{(2,42)}=710.4, p<0.0001$; and Sidak's post hoc test, control vs GPHR ${ }^{F / F}$; GluD2-Cre, $* * p<0.0001$, control vs GPHR ${ }^{F / F} ;$ L7-Cre, $p=0.7015$, GPHR ${ }^{F / F}$;GluD2Cre vs GPHR ${ }^{F / F} ;$ L7-Cre, $\left.* * p<0.0001\right)$. These data suggest that the physical integrity of Pinceau structures depends strongly on the axon status of BCs. Moreover, in GPHR ${ }^{F / F}$;GluD2-Cre mice, Pinceau structures were still observed at P30, whereas they were lost at P60 (Fig. 6B), indicating that Pinceau structures were formed until stabilization of cerebellar circuits.

Double immunostaining for the $\mathrm{GABA}_{\mathrm{A}} \mathrm{R} \alpha 1$ and vGAT to label postsynapses and presynapses, respectively, was performed to investigate whether $\mathrm{BC}$ axons in $G P H R^{F / F}$; GluD2-Cre mice still form synapses with PC soma at P60. $\mathrm{GABA}_{\mathrm{A}} \mathrm{R} \alpha 1$ - and vGAT-immunopositive punctae overlapped almost completely along the soma of PCs in control mice at $\mathrm{P} 60$, displaying the typical pattern of $\mathrm{BC}$ axon terminals (Fig. 6C). In contrast, punctae that were doubly

Table 2. Stereological estimates of total number of the cerebellar PCs (values are in thousands)

\begin{tabular}{llll}
\hline & Animal & GPHR \\
Age & & PC number & GPHR \\
P45 \#1 & & 161.62 & PC number \\
P45 \#2 & & 182.74 & 159.89 \\
P45 \#3 & & 198.85 & 166.71 \\
& Mean & 181.07 & 190.07 \\
& SEM & \pm 10.78 & 172.22 \\
& & & \pm 9.14 \\
Age & & PC number & PC number \\
P60 \#1 & & 161.01 & 133.28 \\
P60 \#2 & & 166.29 & 136.22 \\
P60 \#3 & & 190.51 & 144.99 \\
& Mean & 172.60 & 138.16 \\
& SEM & \pm 9.08 & \pm 3.52 \\
Age & & & \\
P90 \#1 & & PC number & PC number \\
P90 \#2 & & 158.59 & 28.16 \\
P90 \#3 & & 182.45 & 28.31 \\
& Mean & 170.95 & 36.94 \\
& SEM & \pm 7.00 & 31.14 \\
& & & \pm 2.90 \\
\hline
\end{tabular}

positive for $\mathrm{GABA}_{\mathrm{A}} \mathrm{R} \alpha 1$ and vGAT were only sporadically detected in PC somata of GPHR ${ }^{F / F}$;GluD2-Cre mice (Fig. $6 C)$. Unlike BCs, there were no apparent changes in the distribution of stellate cell axon terminals (Fig. 6C). We further performed EM analysis to determine the ultrastructural changes in the AIS and soma of PCs of GPHR ${ }^{F / F}$; GluD2-Cre mice. In control mice, BC axons formed synaptic contacts on the surfaces of PC soma with typical ultrastructural features of asymmetrical synapses, such as synaptic vesicle accumulation in presynaptic terminals, rigid alignment of pre- and postsynaptic partners and electron dense postsynaptic membranes (Fig. 6D-F). However, the PC soma of the GPHR ${ }^{F / F}$;GluD2-Cre mice was predominantly enwrapped with electron-lucent processes of Bergmann glia (BG) instead of synaptic contacts with BCs, suggesting that BG processes filled the space caused by BC degeneration (Fig. 6G-I).

To further examine whether the elimination of BC contacts at PCs is concomitantly accompanied by a decrease in synaptic transmission, we recorded mIPSCs from PCs as a readout of the neurotransmission from BCs. mIPSCs in PCs have been previously classified into two types: small mIPSCs from SCs and large mIPSCs from BCs (Nakayama et al., 2012). The occurrence of large amplitude mIPSCs was dramatically reduced in GPHR ${ }^{F / F}$; GluD2-Cre mice $(n=4)$ when compared with that of control mice $(n=4$; Fig. $7 A, B)$. Accordingly, the mean frequency of large mIPSCs in GPHR ${ }^{F / F}$;GluD2-Cre mice was also significantly lower than that of control mice (Fig. 7C; Table 1; control: $0.44 \pm 0.08 \mathrm{~Hz}, n=4$; $G P H R^{F / F}$; GluD2-Cre: $0.17 \pm 0.07 \mathrm{~Hz}, n=4$; unpaired Student's $t$ test, $\left.t_{(6)}=2.518, * p=0.0454\right)$. Furthermore, the mean amplitude of whole mIPSCs was reduced slightly in GPHR ${ }^{F / F}$;GluD2-Cre mice (Fig. 7D; Table 1; control: 99.9 $\pm 3.6 \mathrm{pA}, n=4$; GPHR ${ }^{\text {F/F }}$;GluD2-Cre: $73.2 \pm 7.8 \mathrm{pA}, n=$ 4; unpaired Student's $t$ test, $\left.t_{(6)}=3.105, * p=0.021\right)$. In contrast, the mean frequencies of small and large amplitude mIPSCs were not different, indicating that the specific reduction of large amplitude events was due to the disruption of BC axons from the PC soma. Taken together, these results indicated that BC synapses on PCs were dramatically reduced, due largely to the axonal degeneration of BCs caused by the dysfunction of Golgi apparatus in $\mathrm{GPHR}^{\mathrm{FIF}}$;GluD2-Cre mice.

\section{Formation of excitatory synapses on PC soma in GPHR ${ }^{F / F}$;GluD2-Cre mice}

Reduced GABAergic transmission from BCs impairs elimination of CF synapses, which leads to abnormal innervation of CF synapses onto PCs (Nakayama et al., 2012). Our morphological and electrophysiological analy- 


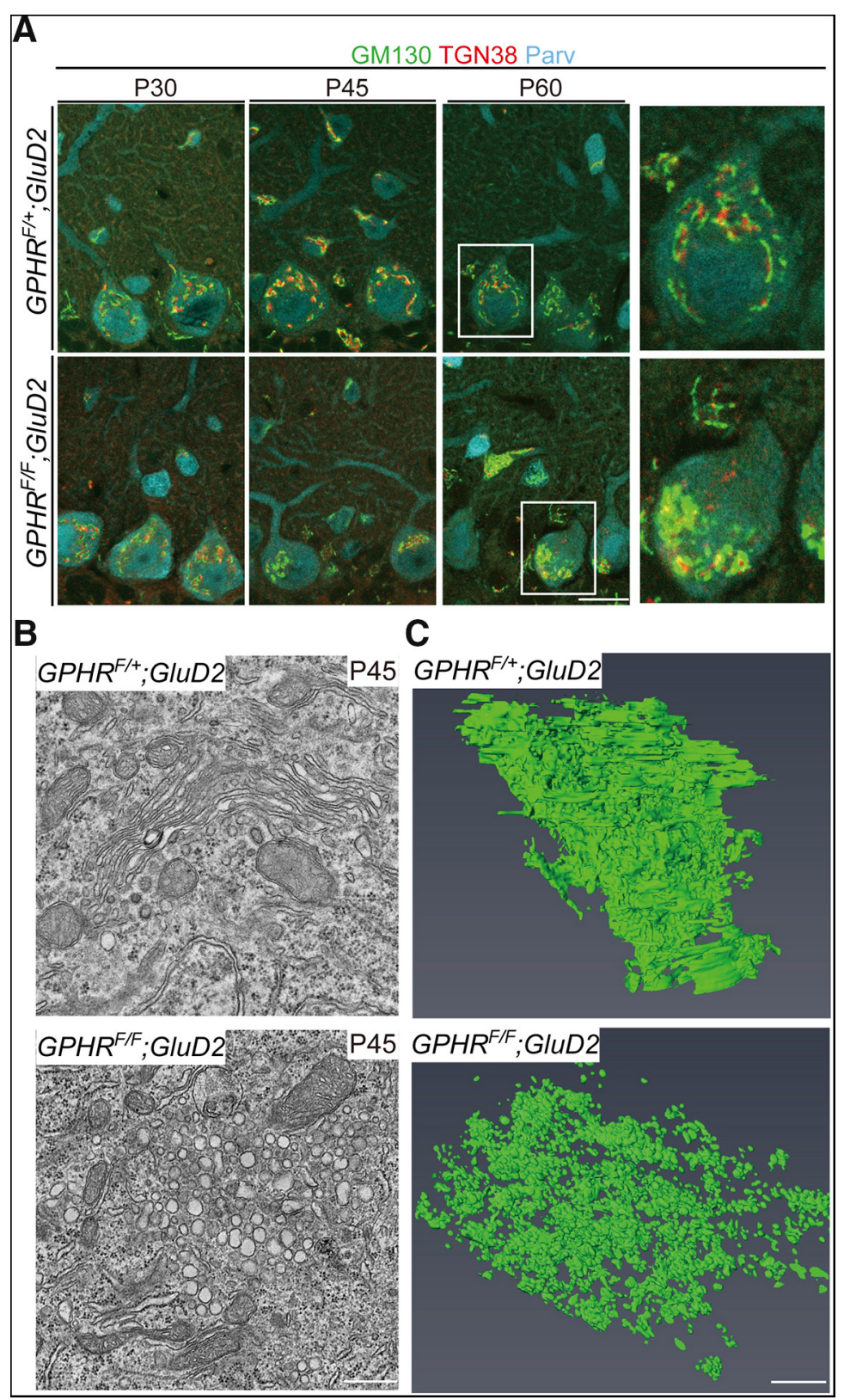

Figure 4. Deletion of GPHR has an effect on the Golgi apparatus organization and distribution in PCs. $\boldsymbol{A}$, Triple immunofluorescence for GM130 (cis-Golgi; green), TGN38 (trans-Golgi; red) and parvalbumin (Parv; cyan) in PCs from GPHR ${ }^{F /+}$;GluD2-Cre and GPHR $R^{F / F}$;GluD2-Cre mice at P30, P45, and P60. Scale bar: $20 \mu \mathrm{m}$. B, Ultrastructural analysis of Golgi apparatus structures in PC somata from GPHR ${ }^{F /+}$;GluD2-Cre and GPHR ${ }^{F / F}$;GluD2-Cre mice at P45. Typical Golgi apparatus structures with flattened cisterns in $G P H R^{F /+}$;GluD2-Cre mice were distinguishable from the Golgi apparatus structures with clusters of vacuoles in GPHR ${ }^{F / F}$;GluD2-Cre mice. $\boldsymbol{C}$, The Golgi apparatus structure reconstructed from serial FIB/SEM images in PCs from GPHR ${ }^{F /+}$;GluD2-Cre and GPHR $R^{F / F}$; GluD2-Cre mice at P45. 3D model shows that fragmentation of the Golgi apparatus occurred in PCs from GPHR ${ }^{F / F}$;GluD2-Cre mice. Scale bars: $500 \mathrm{~nm}$. For $\boldsymbol{A}, \boldsymbol{B}$, three mice per genotype were used; $n=5$ sections per mouse; representative images are shown. See also Movies 1, 2.

ses confirmed the disruption of inhibitory inputs from BCs. Thus, we hypothesized that CF innervation was altered in GPHR ${ }^{F / F}$;GluD2-Cre mice. To test this hypothesis, immunostaining for calbindin and vGlut2, a marker for CF axon terminals, was performed. In normal mice, vGlut2-positive CF terminals were distributed in the molecular layer and associated with PC dendrites after elimination (Miyazaki et al., 2003). In both GPHR ${ }^{F / F} ;$ L7-Cre and control mice, vGlut2-positive CF terminals exclusively innervated spino-dendritic domains in the molecular layer. However, in GPHR ${ }^{F / F}$; GluD2-Cre mice, CF terminals were frequently associated with $\mathrm{PCs}$ soma in addition to dendrites (Fig. 8A, arrowheads). Quantification of the vGlut2 puncta on the PC somata revealed a significant increase in GPHR ${ }^{F / F}$;GluD2-Cre mice when compared with that of control and GPHR ${ }^{F / F} ;$ L7-Cre mice (Table 1; control: 


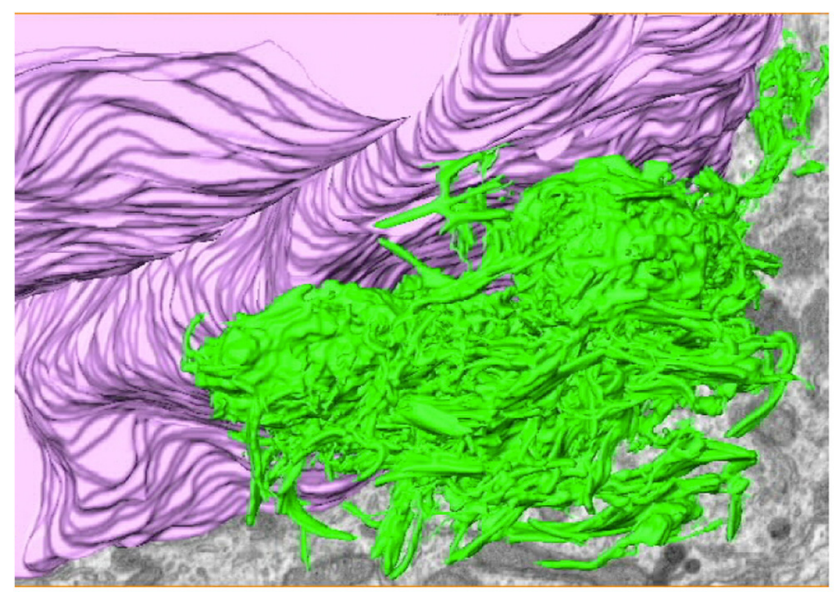

Movie 2. Animation of the three-dimensional reconstructed structure of the Golgi apparatus in a PC of $G P H R^{F /+}$; GluD2-Cre mice at P45. This reconstructed image is obtained from the segmented volume of the serial FIB/SEM images and corresponds to Figure $2 C$, upper panel. Cisternae of the Golgi apparatus and the nucleus are shown by green and magenta, respectively. [View online]

$9.95 \pm 1.07 \% ;$ GPHR ${ }^{F / F} ;$ GluD2-Cre: $69.02 \pm 4.21 \%$; GPHR ${ }^{F / F}$;L7-Cre: $15.06 \pm 1.30 \%$; three mice per group; five sections per mouse; $n=15$ sections for each group; GPHR $^{F /+}$;GluD2-Cre: 172 PCs; GPHR ${ }^{F / F}$; GluD2-Cre: 141 PCs; GPHR ${ }^{F / F}$; L7-Cre: 162 PCs; one-way ANOVA, $F_{(2,42)}$ $=156.4, p<0.0001$; and Sidak's post hoc test, control vs GPHR ${ }^{\text {F/F }}$; GluD2-Cre, **p $<0.0001$, control vs GPHR $^{F / F} ;$ L7-Cre, $p=0.4383$, GPHR ${ }^{F / F}$;GluD2-Cre vs GPHR $R^{F / F}$;L7-Cre, $* * p<0.0001$ ).

Next, to investigate whether somatic spines contacting with CF terminals occur on the surface of PCs in GPHR ${ }^{F / F}$;GluD2-Cre mice, we performed triple immunofluorescence staining for metabotropic glutamate receptor $1 \alpha$ (mGluR1 $\alpha$ ), vGlut2 and calbindin. At the CF-PC synapses, vGlut2 and mGluR1 $\alpha$ are markers for presyn-

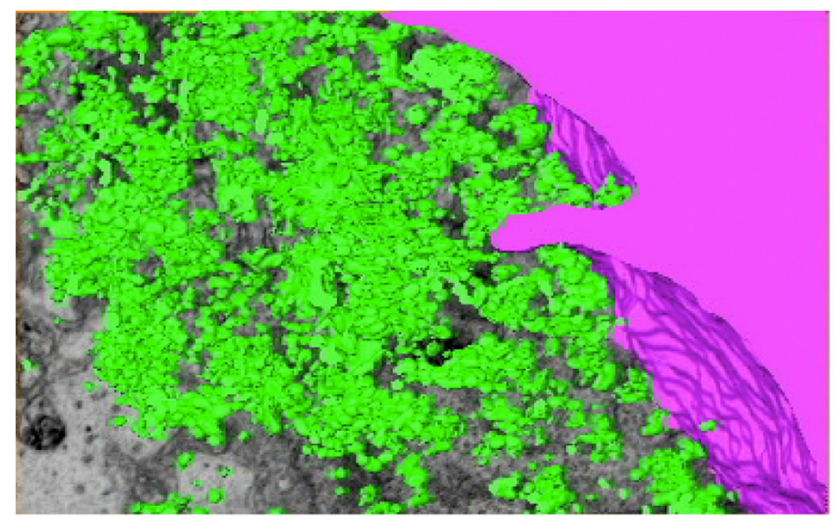

Movie 3. Animation of the three-dimensional reconstructed abnormal structure of the Golgi apparatus in a PC of $G P H R^{F / F}$; GluD2-Cre mice at P45. This reconstructed image is obtained from the segmented volume of the serial FIB/SEM images and corresponds to Figure 2C, lower panel. Vesiculated structures of the Golgi apparatus and the nucleus are shown by green and magenta, respectively. [View online] aptic and postsynaptic proteins, respectively. As hypothesized, mGluR1 $\alpha$-positive punctae overlapped with vGlut2-positive punctae in perisomatic regions of PCs from $G P H R^{F / F}$; GluD2-Cre mice, indicating $C F$ innervation to somatic spines (Fig. 8B). Quantification confirmed that mGluR1 and vGlut2 double-positive puncta on the PC somata were increased significantly in $G P H R^{F / F}$;GluD2-Cre mice when compared with that of control mice (Table 1; GPHR $R^{F /+}$;GluD2-Cre at P60: $9.34 \pm 1.27 \%$; GPHR ${ }^{F / F}$; GluD2-Cre at P60: $45.73 \pm 4.016 \%$; three mice per group; five sections per mouse; $n=15$ sections for each group; GPHR ${ }^{F /+}$;GluD2-Cre at P60: 172 PCs; GPHR ${ }^{F / F}$;GluD2-Cre at P60: 141 PCs; unpaired Student's $t$ test, $t_{(28)}=8.648$, $* p<0.0001)$. In contrast, there was no apparent change in the distribution of vGlut1-positive parallel fiber terminals (Fig. 8C).

Using EM analysis, synaptic contacts on PC somata were observed directly. Some typical BC innervations on PC soma were identified in control and $G P H R^{F / F} ; G / u D 2-C r e$ mice (Fig. 9A,B). Consistent with immunofluorescence analysis (Fig. $8 B$ ), we further observed $C F$ terminals forming synapses with somatic spines of PCs in GPHR ${ }^{F / F}$;GluD2-Cre mice (Fig. 9C,D). Moreover, we frequently observed lamellar bodies, dense stacks of smooth ER, in the perikarya of PCs from $G P H R^{F / F}$;GluD2-Cre mice (Fig. $9 B, E, F$, arrows). These structures contain inositol 1,4,5-triphosphate receptors and become abundant within dendrites in response to stimulation through mGluRs (Yamamoto et al., 1991; Banno and Kohno, 1996). The number of lamellar bodies per $\mathrm{nm}^{2}$ of the perikarya of PCs increased significantly in GPHR ${ }^{F / F}$;GluD2-Cre mice when compared with that of GPHR $^{F /+}$; GluD2-Cre mice (Table 1; control: $0.29 \pm 0.06$, $n=5$; GPHR F/F;GluD2-Cre: $6.49 \pm 0.55, n=5$; unpaired Student's $t$ test, $\left.t_{(8)}=11.27, p<0.0001\right)$. Thus, these data suggest altered intracellular structures because of $C F$ innervation on $\mathrm{PCs}$ soma in GPHR ${ }^{F / F}$;GluD2-Cre mice.

\section{Discussion}

In this study, the crucial role of luminal acidification of the Golgi apparatus in maintaining normal structural integrity of the Golgi apparatus in neurons is reported. Although the relationship between Golgi pathology and neurodegeneration has been suggested based on the genetic analysis of Golgi-related proteins, no studies have thoroughly analyzed abnormalities of cerebellar neuronal circuits on perturbations in luminal pH of the Golgi apparatus in vivo (Alvarez-Miranda et al., 2015). Since available pharmacological blockers lack specificity for manipulation of the luminal $\mathrm{pH}$ of the Golgi apparatus, we believe that genetically modified mouse models are currently an ideal tool to study the relationship between luminal $\mathrm{pH}$ and morphology of the Golgi apparatus.

We demonstrated previously that the luminal $\mathrm{pH}$ of the Golgi apparatus is altered in GPHR mutant Chinese hamster ovary cells (Maeda et al., 2008). In the present study, using a $\mathrm{pH}$-sensitive GFP variant, pHluorin-Golgi, we revealed that the luminal $\mathrm{pH}$ of the Golgi apparatus is elevated in primary cultured neurons that no longer express normal levels of GPHR. Furthermore, it has been reported that the $\mathrm{pH}$ gradient between the ER and the 


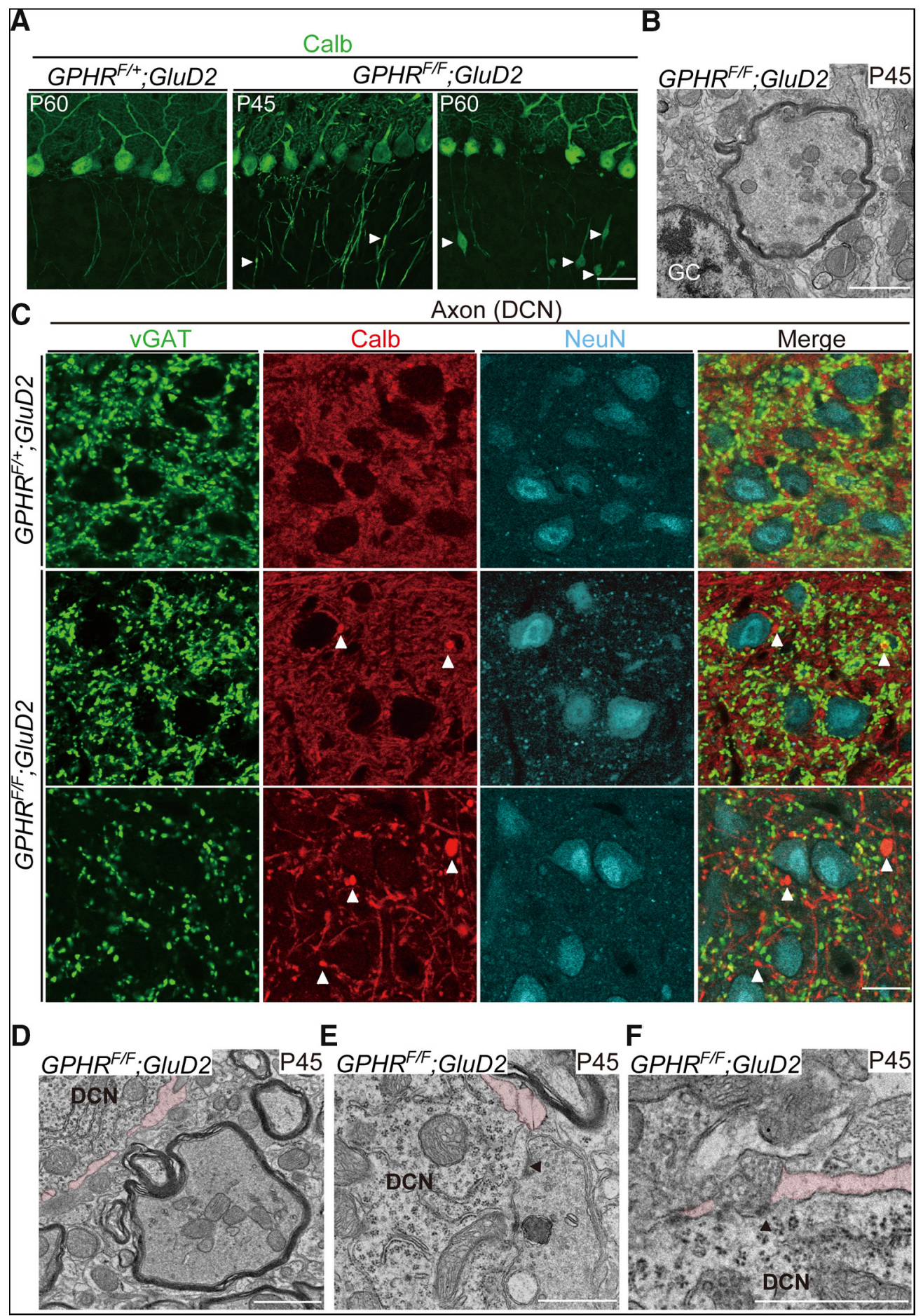

Figure 5. Axonal swelling and abnormal synaptic terminal formation in GPHR-deficient PCs. A, Immunofluorescence for calbindin (Calb; green) in the PC layer of $\mathrm{GPHR}^{F /+}$;GluD2-Cre mice at P60 and GPHR ${ }^{F / F}$;GluD2-Cre mice at P45 and P60. Arrowheads indicate axon swellings. Scale bar: $20 \mu \mathrm{m}$. B. Electron micrographs show axon swelling in the granule cell layer at P45. GC, granule cell. Scale bar: $1 \mu \mathrm{m}$. C, Triple immunofluorescence for vGAT (green), calbindin (Calb; red), and NeuN (cyan) in the DCN of GPHR ${ }^{F /+}$;GluD2-Cre mice at P60 and GPHR ${ }^{F / F}$;GluD2-Cre mice at P45 and P60. PC axon terminal swelling in the DCNs of GPHR ${ }^{F / F}$;GluD2-Cre mice. Arrowheads indicate swellings in the distal axons or axon terminals positive for calbindin. Scale bar: $20 \mu \mathrm{m}$. $\boldsymbol{D}-\boldsymbol{F}$, Electron micrographs showing an axonal swelling in the DCN region $(\boldsymbol{D})$, an abnormal synaptic terminal $(\boldsymbol{E})$, and intercellular spaces (highlighted by pink) on and around the DCN neuron, respectively, at P45. An arrowhead indicates the active zone. Scale bars: $1 \mu \mathrm{m}$. For $\boldsymbol{A}-\boldsymbol{F}$, three mice per genotype were used; $n=5$ sections per mouse; representative images are shown. 


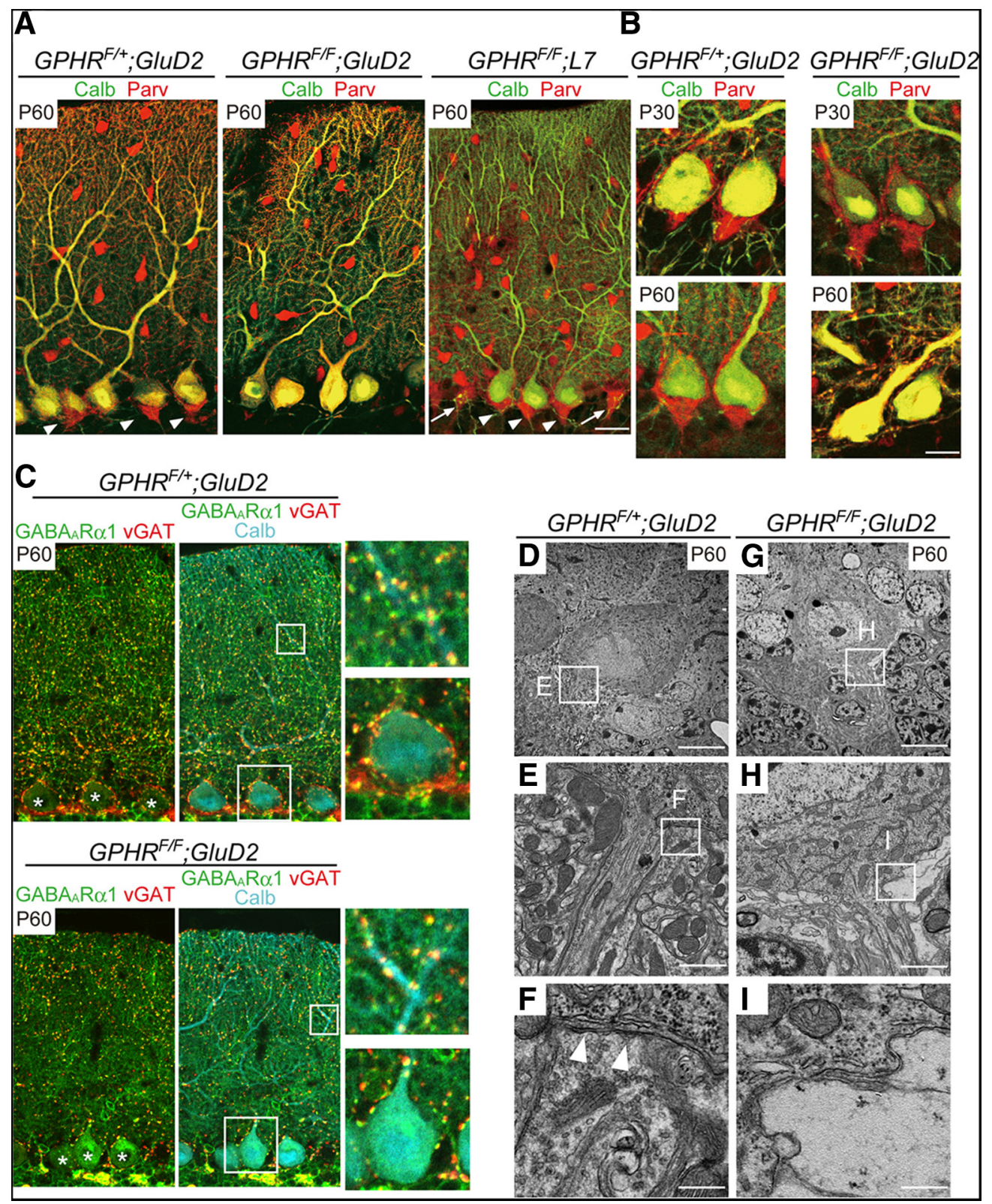

Figure 6. Disappearance of Pinceau structures in GPHR ${ }^{F / F}$;GluD2-Cre mice. A, Double immunofluorescence for calbindin (Calb; green) and parvalbumin (Parv; red) in the PC layer from GPHR ${ }^{F /+}$;GluD2-Cre, GPHR ${ }^{F / F}$;GluD2-Cre, and GPHR ${ }^{F / F} ;$ L7-Cre mice at P60. Arrowheads indicate Pinceau structures. Arrows indicate Pinceau structures without PCs. Scale bar: $20 \mu \mathrm{m}$. $\boldsymbol{B}$, Higher magnification images of Pinceau structures. At P30, the Pinceau structures form cone-shaped structures in both $G P H R^{F /+} ; G / u D 2-C r e$ and $G P H R^{F / F} ; G l u D 2-C r e ~ m i c e$, whereas this structure is absent at P60 for $G P H R^{F / F}$;GluD2-Cre mice. Scale bar: $20 \mu \mathrm{m}$. C, Triple immunofluorescence for GABA $\mathrm{A}_{\mathrm{A}} \mathrm{R}$ (green), vGAT (red) and calbindin (cyan) of the molecular layer at P60. GABA $R \alpha 1$ and vGAT colocalized on PC somata and dendrites in $\mathrm{GPHR}^{F /+}$;GluD2-Cre mice, whereas it decreased in PC somata, but not in the distal dendrites of GPHR/F;GluD2-Cre mice. Boxed regions are enlargements and presented in the right panels. Asterisks indicate PC somata. Scale bar: $20 \mu \mathrm{m}$. Electron micrographs of PC soma (D, $\boldsymbol{G})$ and AIS $(\boldsymbol{E}, \boldsymbol{F}, \boldsymbol{H}, \boldsymbol{I})$ in $G P H R^{F /+}$; GluD2-Cre $(\boldsymbol{D}-\boldsymbol{F})$ and GPHR ${ }^{F / F} ;$ GluD2-Cre mice $(\boldsymbol{G}-\boldsymbol{I})$ at P60. Boxed regions in $\boldsymbol{D}, \boldsymbol{G}$ are enlarged in $\boldsymbol{E}$, $\boldsymbol{H}$, respectively. Boxed regions in $\boldsymbol{E}, \boldsymbol{H}$ are enlarged in $\boldsymbol{F}, \boldsymbol{I}$, respectively. Disruption of $B C$ axon terminal and Bergman glial processes in the Pinceau area of GPHR ${ }^{F / F}$;GluD2-Cre mice $(\boldsymbol{D}-\boldsymbol{F})$. Scale bars: $5 \mu \mathrm{m}(\boldsymbol{A}, \boldsymbol{D}), 1 \mu \mathrm{m}(\boldsymbol{B}, \boldsymbol{E}), 500 \mathrm{~nm}(\boldsymbol{C}, \boldsymbol{F})$. Arrowheads indicate an active zone. For $\boldsymbol{A}-\boldsymbol{I}$, three mice per genotype were used; $n=5$ sections per mouse for each genotype; representative images are shown.

Golgi apparatus disappears by RNA silencing of GPHR in HeLa cells (Vavassori et al., 2013). Taken together, these results support the concept that GPHR is both necessary and sufficient for the maintenance of the Golgi luminal acidic $\mathrm{pH}$ and no other mechanism can compensate for $\mathrm{pH}$ gradient maintenance in neurons.
Our results revealed that loss of GPHR markedly increased the degeneration of PCs. This, in turn, leads to the reduction of cerebellar size and might contribute to the abnormal motor phenotype observed in GPHR ${ }^{F / F}$;GluD2Cre mice. Interestingly, the loss of PCs stands out in the lobule IX. However, this observation is in sharp contrast with 


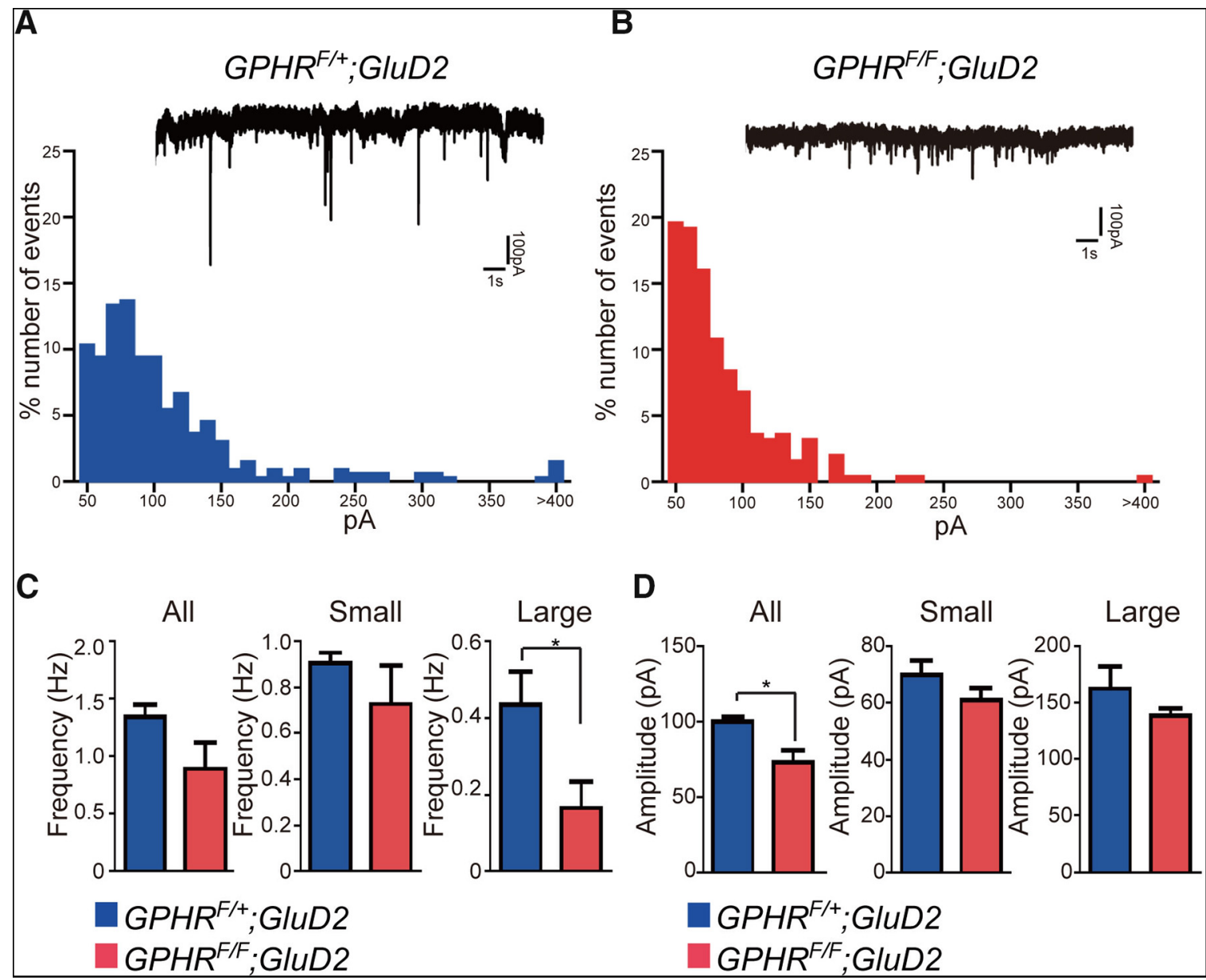

Figure 7. The alteration in GABAergic transmission in PCs from GPHR ${ }^{F / F}$;GluD2-Cre mice. Histogram showing the distribution of mIPSC amplitude in GPHR ${ }^{F /+}$;GluD2-Cre mice $(\boldsymbol{A})$ and GPHR ${ }^{F / F}$;GluD2-Cre mice (B) PCs. ACSF containing $1 \mu \mathrm{M} T \mathrm{TX}$ and $10 \mu \mathrm{M}$ CNQX was used. Insets are representative mIPSC traces. Holding potential $(\mathrm{Vh})=-70 \mathrm{mV}$. $\boldsymbol{C}$, The bar graphs show the average frequency of mIPSC for all, small $(<100 \mathrm{pA})$, and large $(>100 \mathrm{pA})$ events in GPHR ${ }^{F /+}$;GluD2-Cre mice $(n=4)$ and GPHR $R^{F / F}$;GluD2-Cre mice $(n=4)$. Data are presented as the mean \pm SEM; $* p<0.05$ (unpaired Student's $t$ test). $\boldsymbol{D}$, The bar graphs show the average amplitude of mIPSC for all, small $(<100 \mathrm{pA})$, and large $(>100 \mathrm{pA})$ events in GPHR ${ }^{F /+}$;GluD2-Cre mice $(n=4)$ and $G P H R^{F / F}$;GluD2-Cre mice $(n=4)$. Data are presented as the mean \pm SEM; $* p<0.05$ (unpaired Student's $t$ test).

other studies that reported a patterned or area selective PC degeneration (Sarna and Hawkes, 2003; Cerminara et al., 2015). In addition, it has also been reported that lobules IX and $X$ exhibit some level of neuroprotection (Sarna and Hawkes, 2003, 2011). In future, it would be interesting to analyze the factors that confer lobule-dependent neuroprotection in different mouse models.

The observation that PCs begin to degenerate at P60 in $G P H R^{F / F}$;GluD2-Cre mice demonstrates that the luminal acidic $\mathrm{pH}$ of the Golgi apparatus under physiological conditions is essential for PC survival. Impaired luminal acidification of the Golgi apparatus due to deletion of GPHR causes structural disorganization of the Golgi apparatus and results in neurodegeneration. Notably, PCs from GPHR ${ }^{F / F}$;GluD2-Cre mice still exhibit normal organization of the Golgi apparatus with GPHR immunoreactivity until at least P30, indicating that maturation in the early developmental stage of the cerebellum occurs normally in these PCs. In other words, the abolishment of GPHR expression was completed only after stabilization of cer- ebellar circuits. The motor phenotypes because of PC loss in GPHR ${ }^{F / F}$;GluD2-Cre mice were comparable with other mouse models where Golgi-associated proteins such as Rer1 and GM130 are ablated (Liu et al., 2017; Valkova et al., 2017). Loss of GM130 starts from early developmental stages and results in atrophic dendrites and eventual disruption of PCs. This indicates that the proper function of the Golgi apparatus contributes to PC maturation (Liu et al., 2017). However, our current study also suggests that luminal acidification of the Golgi apparatus is also responsible for PC survival after maturation.

Our previous in vitro study showed that GPHR plays an important role in maintaining the morphological integrity of the Golgi apparatus (Maeda et al., 2008). In addition, morphological abnormalities in the Golgi apparatus have also been reported in fibroblasts derived from patients with mutations in the gene encoding ATPVOA2, which is involved in luminal acidification of the Golgi apparatus (Hucthagowder et al., 2009; Foulquier et al., 2012). Consistent with these studies, our morphological analyses 


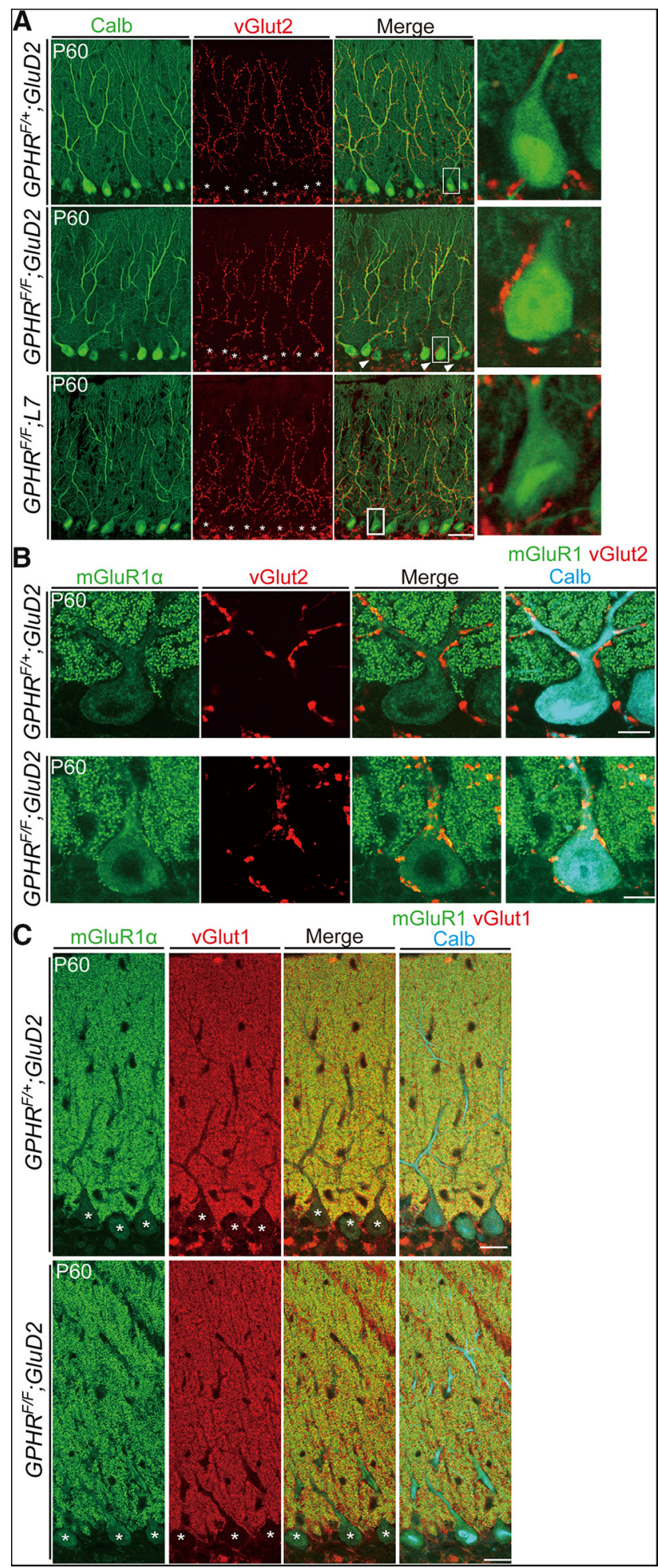

Figure 8. CF terminals innervate $P C$ somata in GPHR ${ }^{F / F}$;GluD2-Cre mice. A, Double immunofluorescence for calbindin (Calb; green) and vGlut2 (red) in the molecular layer at P60. vGlut2-positive CF terminals were observed on PC somata in addition to the dendrites of GPHR ${ }^{F / F}$;GluD2-Cre mice. Arrowheads indicate vGlut2-positive CF terminals on PC soma. Scale bars: $50 \mu \mathrm{m}$. Boxed regions are enlarged and shown in the right panels. $\boldsymbol{B}$, Triple immunofluorescence for mGluR1 $\alpha$ (green), vGlut2 (red), and calbindin (Calb; cyan) in PCs at P60. New

\section{Figure 8. continued}

spines are formed on the surfaces of $P C$ somata in GPHRF/F;GluD2-Cre mice. Asterisks indicate PC somata. Scale bars: $10 \mu \mathrm{m}$. C, Distribution of parallel fiber terminals in GPHR ${ }^{\text {F/F }}$;GluD2-Cre mice. Triple immunofluorescence for mGluR1 $\alpha$ (green), vGlut1 (red), and calbindin (Calb; cyan) in cerebellar sections of $\mathrm{GPHR} \mathrm{R}^{\mathrm{F}+}$;GluD2-Cre mice and GPHR ${ }^{F / F}$;GluD2-Cre mice at P60. Asterisks indicate PC somata. Scale bar: $20 \mu \mathrm{m}$. For $\boldsymbol{A}-\boldsymbol{C}$, three mice per genotype were used; $n=5$ sections per mouse; representative images are shown.

show structural abnormalities of the Golgi apparatus in GPHR-deficient PCs. Interestingly, vesiculation and fragmentation of the Golgi apparatus are pathological hallmarks of various neurodegenerative diseases including amyotrophic lateral sclerosis, Parkinson's, Alzheimer's, and Huntington's diseases (Fan et al., 2008; Haase and Rabouille, 2015; Ayala and Colanzi, 2017). In addition, various models for human neurodegenerative diseases imply that fragmentation of the Golgi apparatus in neurons occurs as a result of the dysregulation of ER-Golgi transport (Huynh et al., 2003; Joshi et al., 2014). Together, our findings suggest that GPHR plays important roles, including morphological maintenance of the Golgi apparatus in neurons. However, genetic screening has not identified GPHR as a causative gene for human diseases. Nonetheless, our present study suggests a causative link between fragmentation of the Golgi apparatus and neurodegeneration. We cannot exclude the possibility that an abnormality of the ER secondarily leads to the abnormal morphology of the Golgi apparatus due to GPHR deficiency. Further studies will be required to elucidate whether transport between ER and the Golgi apparatus is also impaired in GPHR-deficient neurons.

Loss of GM130 and Rer1 proteins in premature PCs affects dendrite and axon morphology, respectively, indicating that the proper function of the Golgi apparatus is important for intact PC polarization (Liu et al., 2017; Valkova et al., 2017). In our study, there was apparently no change in the morphology of PC dendrites in GPHRF/F;GluD2-Cre mice, whereas axonal swelling in GPHR-deficient PCs was observed at P45, and the number and size of axon swellings increased with age. Our observation is in accordance with those from previous studies in mouse models where PCs lack Golgiassociated proteins (Watanabe et al., 2010; Valkova et al., 2017). However, it is unlikely that the axonal swellings in GPHR-deficient PCs are related to myelin abnormality as observed in PCs deficient in ceramide glucosyltransferase, a Golgi-associated enzyme (Watanabe et al., 2010), because myelination of GPHR-deficient PC axons appears normal. Furthermore, we also noticed that a larger portion of the soma of DCN neurons of GPHR ${ }^{F / F}$;GluD2-Cre mice were surrounded with intercellular spaces because of the loss of axosomatic synapses as early as P45. Importantly, the synaptic terminals of BCs in GPHRF/F;GluD2-Cre mice were also lost at P60, which strengthens our notion that impairment of luminal acidification of the Golgi apparatus is closely associated with axonal and synaptic pathology. Axonal swelling is a hall- 

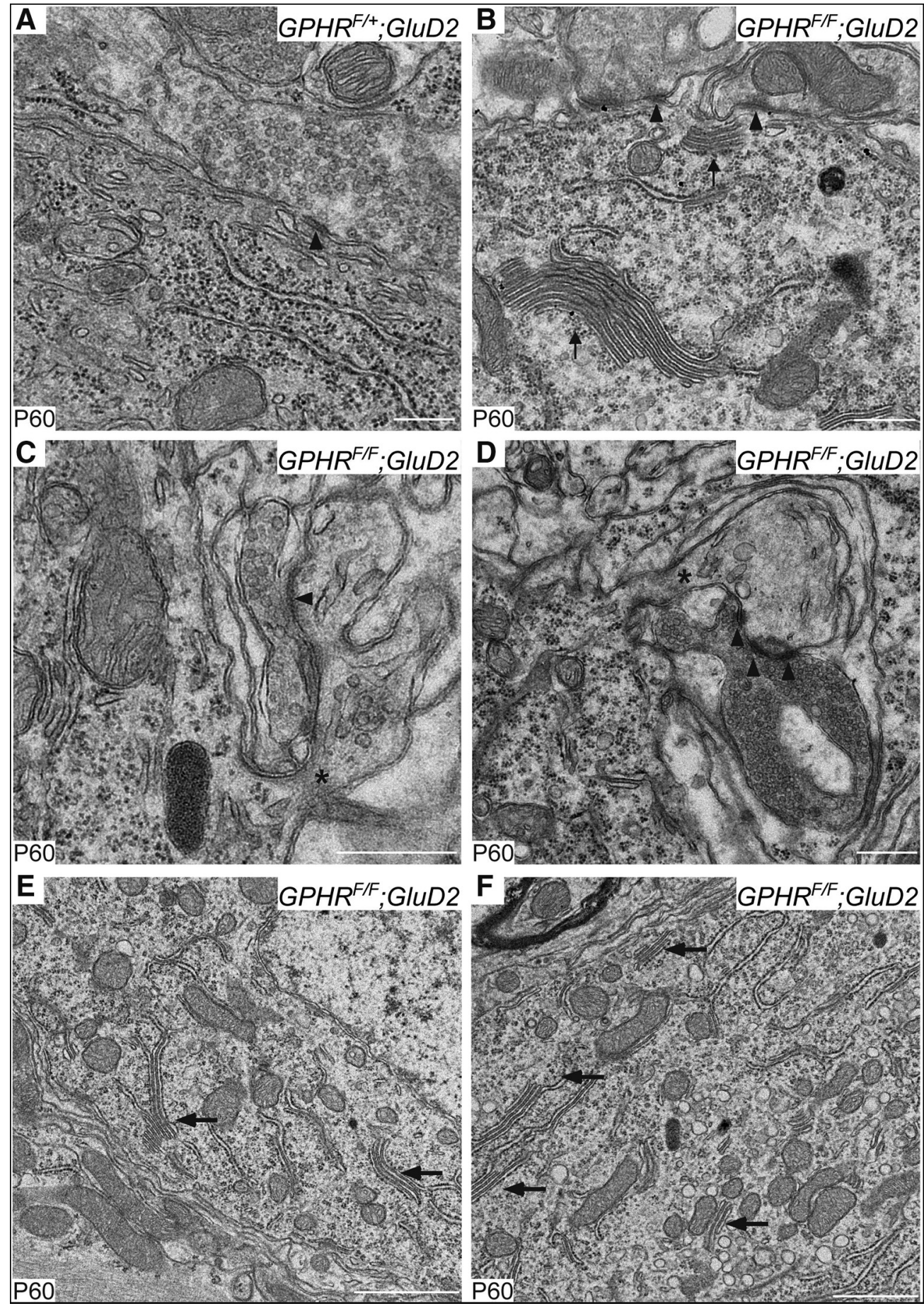

Figure 9. CF innerved to PC soma in GPHR ${ }^{F / F}$;GluD2-Cre mice. Electron micrographs of PC soma in $G P H R^{F /+} ; G / u D 2-C r e(A)$ and $G P H R^{F / F} ; G l u D 2-C r e$ mice $(\boldsymbol{B}-\boldsymbol{F})$ at P60. Arrowheads indicate active zones. Arrows indicate dense stacks of the cisternae of the smooth endoplasmic reticulum. Asterisks indicate necks of the spine from PC soma. Scale bars: $500 \mathrm{~nm}$. $\boldsymbol{A}-\boldsymbol{F}$, Three mice per genotype were used; $n=5$ sections per mouse; representative images are shown.

mark of axonal dystrophy, which can lead to neurodegeneration (Coleman, 2005). Major causes for axon degeneration converge in the dysfunctions of axonal transport, the autophagy-lysosome system, mitochondria, and increased intra-axonal calcium (Coleman, 2005; Komatsu et al., 2007; Nishiyama et al., 2007; Liang et al., 2010; Koike et al., 2017; Yamaguchi et al., 2017). Al- though the direct link between impairment of luminal acidification of the Golgi apparatus and axon and synaptic abnormalities remains unclear, it is possible that loss of GPHR is associated with axonal transport dysfunction.

Intriguingly, CF innervation on PC soma has been observed in PC-specific deletion of GluD2 and RORa because of territorial changes in parallel fibers and CFs 
(Miyazaki et al., 2010; Chen et al., 2013). Furthermore, reduced GABAergic transmission from BCs leads to abnormal elimination of CF synapses onto PCs during early developmental stages (Nakayama et al., 2012). However, the role of GABAergic transmission in maintaining proper CF innervation in the adult cerebellum has not been examined. In our mouse model, CF innervates the PC soma instead of the BC axonal terminal territory after stabilization of the cerebellar circuits. This novel finding suggests that, in addition to heterosynaptic competition of excitatory innervations, the inhibitory-excitatory imbalance can also result in a redistribution of the innervation territory on mature PCs. Further studies are required to understand the roles of $\mathrm{BCs}$ in maintenance of proper $\mathrm{CF}$ innervation onto PCs by use of BC-specific Cre mouse lines.

What could be the pathological consequences of altered cerebellar neuronal circuitry in GPHR ${ }^{F / F}$;GluD2-Cre mice? Our morphological data showed that the formation of somatic spines in GPHR-deficient PCs and the targeting of CFs to these somatic spines as well as the electrophysiological data suggesting disrupted and nonfunctional inhibitory BCs hint at a possible excitatory-inhibitory imbalance in these PCs. Unlike the normal situation, where CF inputs are created only in dendrites, the direct excitation of the soma by CF implies that voltage changes are minimal and thus result in a dramatic increase in the PC membrane potential. Moreover, the severity of depolarization around the PC soma is maintained because inhibition from BC terminals no longer exists. As calcium permeable channels open because of depolarized membrane potentials, overexcitation may create an unnecessarily large calcium load in the PC cytoplasm, leading to calcium excitotoxicity. This may lead to the eventual disruption and death of PCs. Indeed, PCs from GPHRF/F;GluD2-Cre mice possess dense stacks of smooth $\mathrm{ER}$, which prevent excessive $\mathrm{Ca}^{2+}$ release from the reservoir (Banno and Kohno, 1996) and respond to calcium excitotoxicity because of the excitatory-inhibitory imbalance. Nevertheless, GPHR-deficient PCs are largely lost with age, suggesting that neurodegeneration is likely caused by impairment of luminal acidification of the Golgi apparatus. Further studies must be conducted to elucidate the function of the Golgi apparatus for maintaining the integrity of neuronal circuits.

\section{References}

Alvarez-Miranda EA, Sinnl M, Farhan H (2015) Alteration of Golgi structure by stress: a link to neurodegeneration? Front Neurosci 9:435.

Ango $\mathrm{F}$, di Cristo G, Higashiyama $\mathrm{H}$, Bennett V, Wu P, Huang ZJ (2004) Ankyrin-based subcellular gradient of neurofascin, an immunoglobulin family protein, directs GABAergic innervation at Purkinje axon initial segment. Cell 119:257-272.

Ayala I, Colanzi A (2017) Alterations of Golgi organization in Alzheimer's disease: a cause or a consequence? Tissue Cell 49:133140.

Baloyannis SJ (2014) Golgi apparatus and protein trafficking in Alzheimer's disease. J Alzheimers Dis 42 [Suppl 3]:S153-S162.

Banno T, Kohno K (1996) Conformational changes of smooth endoplasmic reticulum induced by brief anoxia in rat Purkinje cells. $J$ Comp Neurol 369:462-471.

Campenot RB, Soin J, Blacker M, Lund K, Eng H, MacInnis BL (2003) Block of slow axonal transport and axonal growth by brefeldin A in compartmented cultures of rat sympathetic neurons. Neuropharmacology 44:1107-1117.

Cerminara NL, Lang EJ, Sillitoe RV, Apps R (2015) Redefining the cerebellar cortex as an assembly of non-uniform Purkinje cell microcircuits. Nat Rev Neurosci 16:79-93.

Chen XR, Heck N, Lohof AM, Rochefort C, Morel MP, Wehrle R, Doulazmi M, Marty S, Cannaya V, Avci HX, Mariani J, Rondi-Reig L, Vodjdani G, Sherrard RM, Sotelo C, Dusart I (2013) Mature Purkinje cells require the retinoic acid-related orphan receptor- $\alpha$ $(\mathrm{ROR} \alpha)$ to maintain climbing fiber mono-innervation and other adult characteristics. J Neurosci 33:9546-9562.

Chintawar S, Hourez R, Ravella A, Gall D, Orduz D, Rai M, Bishop DP, Geuna S, Schiffmann SN, Pandolfo M (2009) Grafting neural precursor cells promotes functional recovery in an SCA1 mouse model. J Neurosci 29:13126-13135.

Chintawar S, Fregnan F, Pandolfo M, Geuna S (2012) Stereological quantification of cerebellar Purkinje cells: literature review and description of a variation of the physical disector method adapted to confocal laser microscopy. Neuroquantology 10:125-131.

Climer LK, Dobretsov M, Lupashin V (2015) Defects in the COG complex and COG-related trafficking regulators affect neuronal Golgi function. Front Neurosci 9:405.

Coleman M (2005) Axon degeneration mechanisms: commonality amid diversity. Nat Rev Neurosci 6:889-898.

Condon KH, Ho J, Robinson CG, Hanus C, Ehlers MD (2013) The Angelman syndrome protein Ube3a/E6AP is required for Golgi acidification and surface protein sialylation. J Neurosci 33:37993814.

Demaegd D, Foulquier F, Colinet AS, Gremillon L, Legrand D, Mariot P, Peiter E, Van Schaftingen E, Matthijs G, Morsomme P (2013) Newly characterized Golgi-localized family of proteins is involved in calcium and $\mathrm{pH}$ homeostasis in yeast and human cells. Proc Natl Acad Sci USA 110:6859-6864.

Fan J, Hu Z, Zeng L, Lu W, Tang X, Zhang J, Li T (2008) Golgi apparatus and neurodegenerative diseases. Int $\mathrm{J}$ Dev Neurosci 26:523-534.

Foulquier F, Amyere M, Jaeken J, Zeevaert R, Schollen E, Race V, Bammens R, Morelle W, Rosnoblet C, Legrand D, Demaegd D, Buist N, Cheillan D, Guffon N, Morsomme P, Annaert W, Freeze HH, Van Schaftingen E, Vikkula M, Matthijs G (2012) TMEM165 deficiency causes a congenital disorder of glycosylation. Am J Hum Genet 91:15-26.

Franklin KBJ, Paxinos G (2007) The mouse brain in stereotaxic coordinates, Ed 3. New York, NY: Academic Press.

Gianola S, Savio T, Schwab ME, Rossi F (2003) Cell-autonomous mechanisms and myelin-associated factors contribute to the development of Purkinje axon intracortical plexus in the rat cerebellum. J Neurosci 23:4613-4624.

Gonatas NK, Stieber A, Mourelatos Z, Chen Y, Gonatas JO, Appel SH, Hays AP, Hickey WF, Hauw JJ (1992) Fragmentation of the Golgi apparatus of motor neurons in amyotrophic lateral sclerosis. Am J Pathol 140:731-737.

Guyenet SJ, Furrer SA, Damian VM, Baughan TD, Spada Ar L, Garden GA (2010) A simple composite phenotype scoring system for evaluating mouse models of cerebellar ataxia. J Vis Exp 39: e1787.

Haase G, Rabouille C (2015) Golgi fragmentation in ALS motor neurons. New mechanisms targeting microtubules, tethers, and transport vesicles. Front Neurosci 9:448.

Horton AC, Rácz B, Monson EE, Lin AL, Weinberg RJ, Ehlers MD (2005) Polarized secretory trafficking directs cargo for asymmetric dendrite growth and morphogenesis. Neuron 48:757-771.

Hucthagowder V, Morava E, Kornak U, Lefeber DJ, Fischer B, Dimopoulou A, Aldinger A, Choi J, Davis EC, Abuelo DN, Adamowicz M, Al-Aama J, Basel-Vanagaite L, Fernandez B, Greally MT, Gillessen-Kaesbach G, Kayserili H, Lemyre E, Tekin M, Türkmen S, et al. (2009) Loss-of-function mutations in ATP6V0A2 impair vesicular trafficking, tropoelastin secretion and cell survival. Hum Mol Genet 18:2149-2165. 
Huynh DP, Yang HT, Vakharia H, Nguyen D, Pulst SM (2003) Expansion of the polyQ repeat in ataxin-2 alters its Golgi localization, disrupts the Golgi complex and causes cell death. Hum Mol Genet $12: 1485-1496$.

Ip CW, Cheong D, Volkmann J (2017) Stereological estimation of dopaminergic neuron number in the mouse substantia nigra using the optical fractionator and standard microscopy equipment. J Vis Exp 127:e1787.

Joshi G, Chi Y, Huang Z, Wang Y (2014) $A \beta$-induced Golgi fragmentation in Alzheimer's disease enhances $A \beta$ production. Proc Natl Acad Sci USA 111:E1230-E1239.

Kamikubo Y, Shimomura T, Fujita Y, Tabata T, Kashiyama T, Sakurai T, Fukurotani K, Kano M (2013) Functional cooperation of metabotropic adenosine and glutamate receptors regulates postsynaptic plasticity in the cerebellum. J Neurosci 33:18661-18671.

Kellokumpu S, Sormunen R, Kellokumpu I (2002) Abnormal glycosylation and altered Golgi structure in colorectal cancer: dependence on intra-Golgi pH. FEBS Lett 516:217-224.

Klumperman $J$ (2011) Architecture of the mammalian Golgi. Cold Spring Harb Perspect Biol 3

Koike M, Shibata M, Sunabori T, Yamaguchi J, Sakimura K, Komatsu M, Tanaka K, Uchiyama Y (2017) Purkinje cells are more vulnerable to the specific depletion of cathepsin D than to that of Atg7. Am J Pathol 187:1586-1600.

Komatsu M, Wang QJ, Holstein GR, Friedrich VL Jr, Iwata J, Kominami E, Chait BT, Tanaka K, Yue Z (2007) Essential role for autophagy protein Atg7 in the maintenance of axonal homeostasis and the prevention of axonal degeneration. Proc Natl Acad Sci USA 104:14489-14494.

Kornak U, Reynders E, Dimopoulou A, van Reeuwijk J, Fischer B, Rajab A, Budde B, Nürnberg P, Foulquier F, Lefeber D, Urban Z, Gruenewald S, Annaert W, Brunner HG, van Bokhoven H, Wevers R, Morava E, Matthijs G, Van Maldergem L, Mundlos S (2008) Impaired glycosylation and cutis laxa caused by mutations in the vesicular H+-ATPase subunit ATP6V0A2. Nat Genet 40:32-34.

Liang CC, Wang C, Peng X, Gan B, Guan JL (2010) Neural-specific deletion of FIP200 leads to cerebellar degeneration caused by increased neuronal death and axon degeneration. J Biol Chem 285:3499-3509.

Liu C, Mei M, Li Q, Roboti P, Pang Q, Ying Z, Gao F, Lowe M, Bao $S$ (2017) Loss of the golgin GM130 causes Golgi disruption, Purkinje neuron loss, and ataxia in mice. Proc Natl Acad Sci USA 114:346-351.

Maeda Y, Ide T, Koike M, Uchiyama Y, Kinoshita T (2008) GPHR is a novel anion channel critical for acidification and functions of the Golgi apparatus. Nat Cell Biol 10:1135-1145.

Matsuki T, Matthews RT, Cooper JA, van der Brug MP, Cookson MR, Hardy JA, Olson EC, Howell BW (2010) Reelin and stk25 have opposing roles in neuronal polarization and dendritic Golgi deployment. Cell 143:826-836.

Miesenböck G, De Angelis DA, Rothman JE (1998) Visualizing secretion and synaptic transmission with $\mathrm{pH}$-sensitive green fluorescent proteins. Nature 394:192-195.

Miyazaki T, Fukaya M, Shimizu H, Watanabe M (2003) Subtype switching of vesicular glutamate transporters at parallel fibrePurkinje cell synapses in developing mouse cerebellum. Eur $\mathrm{J}$ Neurosci 17:2563-2572.

Miyazaki T, Yamasaki M, Takeuchi T, Sakimura K, Mishina M, Watanabe M (2010) Ablation of glutamate receptor GluR $\delta 2$ in adult Purkinje cells causes multiple innervation of climbing fibers by inducing aberrant invasion to parallel fiber innervation territory. $\mathrm{J}$ Neurosci 30:15196-15209.

Mourelatos Z, Gonatas NK, Stieber A, Gurney ME, Dal Canto MC (1996) The Golgi apparatus of spinal cord motor neurons in transgenic mice expressing mutant $\mathrm{Cu}, \mathrm{Zn}$ superoxide dismutase becomes fragmented in early, preclinical stages of the disease. Proc Natl Acad Sci USA 93:5472-5477.

Nakayama H, Miyazaki T, Kitamura K, Hashimoto K, Yanagawa $Y$, Obata K, Sakimura K, Watanabe M, Kano M (2012) GABAergic inhibition regulates developmental synapse elimination in the cerebellum. Neuron 74:384-396.

Nishiyama J, Miura E, Mizushima N, Watanabe M, Yuzaki M (2007) Aberrant membranes and double-membrane structures accumulate in the axons of Atg5-null Purkinje cells before neuronal death. Autophagy 3:591-596.

Palay SL, Chan-Palay V (1974) Cerebellar cortex. New York, NY: Springer.

Palokangas H, Metsikkö K, Väänänen K (1994) Active vacuolar $\mathrm{H}+$ ATPase is required for both endocytic and exocytic processes during viral infection of BHK-21 cells. J Biol Chem 269:17577-17585.

Papanikou E, Glick BS (2014) Golgi compartmentation and identity. Curr Opin Cell Biol 29:74-81.

Saito H, Tsumura H, Otake S, Nishida A, Furukawa T, Suzuki N (2005) L7/Pcp-2-specific expression of Cre recombinase using knock-in approach. Biochem Biophys Res Commun 331:1216-1221.

Sakaguchi T, Leser GP, Lamb RA (1996) The ion channel activity of the influenza virus M2 protein affects transport through the Golgi apparatus. J Cell Biol 133:733-747.

Sarna JR, Hawkes R (2003) Patterned Purkinje cell death in the cerebellum. Prog Neurobiol 70:473-507.

Sarna JR, Hawkes R (2011) Patterned Purkinje cell loss in the ataxic sticky mouse. Eur J Neurosci 34:79-86.

Schneider CA, Rasband WS, Eliceiri KW (2012) NIH Image to ImageJ: 25 years of image analysis. Nat Methods 9:671-675.

Sotelo C (2008) Development of "Pinceaux" formations and dendritic translocation of climbing fibers during the acquisition of the balance between glutamatergic and gamma-aminobutyric acidergic inputs in developing Purkinje cells. J Comp Neurol 506:240-262.

Tanida I, Ueno T, Uchiyama Y (2014) A super-ecliptic, pHluorinmKate2, tandem fluorescent protein-tagged human LC3 for the monitoring of mammalian autophagy. PLoS One 9:e110600.

Tartakoff AM (1983) Perturbation of vesicular traffic with the carboxylic ionophore monensin. Cell 32:1026-1028.

Tarutani M, Nakajima K, Uchida Y, Takaishi M, Goto-Inoue N, Ikawa M, Setou M, Kinoshita T, Elias PM, Sano S, Maeda Y (2012) GPHR-dependent functions of the Golgi apparatus are essential for the formation of lamellar granules and the skin barrier. J Invest Dermatol 132:2019-2025.

Valkova C, Liebmann L, Krämer A, Hübner CA, Kaether C (2017) The sorting receptor Rer1 controls Purkinje cell function via voltage gated sodium channels. Sci Rep 7:41248.

Vavassori S, Cortini M, Masui S, Sannino S, Anelli T, Caserta IR, Fagioli C, Mossuto MF, Fornili A, van Anken E, Degano M, Inaba K, Sitia R (2013) A pH-regulated quality control cycle for surveillance of secretory protein assembly. Mol Cell 50:783-792.

Watanabe S, Endo S, Oshima E, Hoshi T, Higashi H, Yamada K, Tohyama K, Yamashita T, Hirabayashi Y (2010) Glycosphingolipid synthesis in cerebellar Purkinje neurons: roles in myelin formation and axonal homeostasis. Glia 58:1197-1207.

Weisz OA (2003) Acidification and protein traffic. Int Rev Cytol 226: 259-319.

Xu M, Ouyang Q, Gong J, Pescosolido MF, Pruett BS, Mishra S, Schmidt M, Jones RN, Gamsiz Uzun ED, Lizarraga SB, Morrow EM (2018) Mixed neurodevelopmental and neurodegenerative pathology in Nhe6-null mouse model of christianson syndrome. eNeuro 4.

Yamaguchi J, Suzuki C, Nanao T, Kakuta S, Ozawa K, Tanida I, Saitoh T, Sunabori T, Komatsu M, Tanaka K, Aoki S, Sakimura K, Uchiyama Y (2017) Atg9a deficiency causes axon-specific lesions including neuronal circuit dysgenesis. Autophagy 1-14. [CrossRef] Yamamoto A, Otsu H, Yoshimori T, Maeda N, Mikoshiba K, Tashiro Y (1991) Stacks of flattened smooth endoplasmic reticulum highly enriched in inositol 1,4,5-trisphosphate (InsP3) receptor in mouse cerebellar Purkinje cells. Cell Struct Funct 16:419-432.

Yamasaki M, Miyazaki T, Azechi H, Abe M, Natsume R, Hagiwara T, Aiba A, Mishina M, Sakimura K, Watanabe M (2011) Glutamate receptor $\delta 2$ is essential for input pathway-dependent regulation of synaptic AMPAR contents in cerebellar Purkinje cells. J Neurosci 31:3362-3374. 